\title{
Poly(lactic acid) Composites Containing Carbon-Based Nanomaterials: A Review
}

\author{
Carolina Gonçalves ${ }^{1}$ (D), Inês C. Gonçalves ${ }^{2,3}$, Fernão D. Magalhães ${ }^{1}$ and Artur M. Pinto ${ }^{1,2,3, *}$ \\ 1 LEPABE-Faculdade de Engenharia, Universidade do Porto, rua Dr. Roberto Frias, 4200-465 Porto, Portugal; \\ carol.goncalves8827@gmail.com (C.G.); fdmagalh@fe.up.pt (F.D.M.) \\ 2 INEB-National Institute of Biomedical Engineering, University of Porto, Rua do Campo Alegre, 823, \\ 4150-180 Porto, Portugal; icastro@ineb.up.pt \\ 3 i3S-Institute for Innovation and Health Research, University of Porto, Rua Alfredo Allen, 208, \\ 4200-135 Porto, Portugal \\ * Correspondence: arturp@fe.up.pt; Tel.: +351-22-508-1400
}

Academic Editor: Alexander Böker

Received: 15 June 2017; Accepted: 4 July 2017; Published: 6 July 2017

\begin{abstract}
Poly(lactic acid) (PLA) is a green alternative to petrochemical commodity plastics, used in packaging, agricultural products, disposable materials, textiles, and automotive composites. It is also approved by regulatory authorities for several biomedical applications. However, for some uses it is required that some of its properties be improved, namely in terms of thermo-mechanical and electrical performance. The incorporation of nanofillers is a common approach to attain this goal. The outstanding properties of carbon-based nanomaterials $(\mathrm{CBN})$ have caused a surge in research works dealing with PLA/CBN composites. The available information is compiled and reviewed, focusing on PLA/CNT (carbon nanotubes) and PLA/GBM (graphene-based materials) composites. The production methods, and the effects of CBN loading on PLA properties, namely mechanical, thermal, electrical, and biological, are discussed.
\end{abstract}

Keywords: PLA; graphene-based materials; carbon nanotubes; composites; mechanical properties; thermal properties; electrical properties; biological properties

\section{Introduction}

The growing environmental awareness and new rules and regulations are forcing the industries to seek more ecologically friendly materials for their products [1]. In the last two decades, industrial and academic research on polymer composites was pursued to provide added value properties to the neat polymer without sacrificing its processability or adding excessive weight [2].

Poly(lactic acid) (PLA), which is derived from natural sources, biodegradable, and bioabsorbable, has had significant demand due to presenting versatile applications in packaging, pharmaceutical, textiles, engineering, chemical industries, automotive composites, biomedical and tissue engineering fields [3]. Its biodegradation time can be tuned, depending on the molecular weight, crystallinity, and material geometry [4]. However, the relatively low glass transition temperature, low thermal dimensional stability, and mechanical ductility limit the number of its applications. A significant body of research has dealt with the use of fillers for improving the properties of PLA [5-7]. In this context, carbon based nanomaterials (CBN), offer the potential to combine PLA properties with several of their unique features, such as high mechanical strength, electrical conductivity, thermal stability and bioactivity [8-16]. Carbon nanotubes (CNT) and graphene-based materials (GBM) are state of the art and very promising representatives of these materials. CNT have exceptional mechanical properties, aspect ratio, electrical and thermal conductivities, and chemical stability. However, their production methods are usually more complex and expensive, often leaving toxic metal residues [17-20]. Hence, 
GBM provide an alternative option to produce functional composites due to their excellent properties and the natural abundance of their precursor, graphite. Moreover, GBM can be produced by simple and inexpensive physico-chemical methods [21-24].

In the last years there has been a surge of research works on PLA/CNT and PLA/GBM composites. Due to the large amount of information available, there is the need to congregate, compare and withdraw conclusions.

Several recent reviews have addressed PLA [3,25-30] and CBN [30-46] production, applications and properties, however, none of these focus on PLA/CBN composites. This work presents a comprehensive review on the current knowledge regarding the production of PLA/CBN composites and the resulting properties, namely mechanical, electrical, thermal and biological.

\section{Poly(lactic acid) (PLA)}

PLA is a thermoplastic aliphatic polyester commonly produced by direct condensation polymerization of lactic acid or by ring-opening polymerization of lactide. As lactic acid is a chiral molecule, existing in $\mathrm{L}$ and $\mathrm{D}$ isomers, the term "poly(lactic acid)" refers to a family of polymers: poly-L-lactic acid (PLLA), poly-D-lactic acid (PDLA), and poly-D,L-lactic acid (PDLLA). The 2 optically active configurations of lactic acid, the $\mathrm{L}(+)$ and $\mathrm{D}(-)$ stereoisomers are produced, respectively by bacterial homo- or hetero-fermentation of carbohydrates. A great variety of carbohydrate sources can be used to produce lactic acid, like molasses, corn syrup, whey, dextrose, and cane or beet sugar. Nowadays, industry only uses the fermentation process, because the synthetic routes have major limitations, as the inability of selective production of the L-lactic acid stereoisomer, and high manufacturing costs $[47,48]$.

PLA can be polymerized by diverse methods, like polycondensation, ring opening polymerization, azeotropic dehydration condensation, and enzymatic polymerization. Direct polymerization and ring opening polymerization are the most used. Controlling polymerization parameters is important, since PLA properties vary with isomer composition, temperature, and reaction time used [3,25,28,29,48-51].

Increasing interest in PLA is related to some characteristics that are lacking in other polymers, namely regarding renewability, biocompatibility, processability, and energy saving [29]. PLA is derived from renewable and biodegradable resources, and its degradation products are non-pollutant and non-toxic. Thus, PLA is a green alternative to petrochemical commodity plastics, used in packaging, agricultural products, disposable materials, textiles, and automotive [25]. Furthermore, PLA has several bioapplications, such as biodegradable matrix for surgical implants, and in drug delivery systems [3].

The use of PLA has some shortcomings, related to poor chemical modifiability (absence of readily reactive side-chain groups), mechanical ductility [50], and relatively high price [28]. To overcome some of these issues, some approaches are commonly used, like blending with other polymers [52-59], functionalization [60-64], and addition of nanofillers [6,7,48,65-70]. The last is an interesting approach, since with small filler amounts it is possible to enhance desired features, keeping PLA's key properties intact. The most used nanofillers are nanoclays [5,71-80], nanosilicas $[6,68,69,73,81,82]$, and carbon nanomaterials $[7,77,83-88]$.

\section{Carbon-Based Nanomaterials (CBN)}

There are several types of carbon-based nanomaterials (carbon nanotubes, graphene-based materials, fullerenes, nanodiamonds) and most have been tested to improve PLA properties. This review is focused on the most widely tested and available: CNT and GBM. The high specific area of these materials allows for low loadings to be sufficient to tune key properties concerning mechanical, thermal, electrical, and biological performance. 


\section{CBN Production Methods and Modifications}

Graphene is the elementary structure of graphite, being a one carbon atom thick sheet, composed of $\mathrm{sp}^{2}$ carbon atoms arranged in a flat honeycomb structure composed of two equivalent sub-lattices of carbon atoms bonded together with $\sigma$ bonds (in plane) and a $\pi$ bond (out-of-plane), which contributes to a delocalized network of electrons $[39,46,89]$. These unique characteristics explain its unmatched electronic, mechanical, optical and thermal properties. For that reason, this material has been studied to be applied in many fields, such as electronics [90-95], energy [96-99], membrane [100-103], composite [21,22,24,104], and biomedical technology [11,105-107].

The intrinsic properties of graphene, and GBM in general, are affected by the production or modification methods. For example, structural integrity of graphene sheets is disrupted by oxidation and some other chemical modifications. The dimensions (diameter and thickness) of the final GBM also depend on the raw materials and methods employed [11,34,35,46,90]. Thus, those should be chosen according to desired applications.

GBM can be obtained by top-down and bottom-up approaches [104]. The first involves exfoliating graphite to obtain few or single layer graphene sheets $[38,108]$. The second, consists in assembling graphene from deposition of carbon atoms from other sources $[109,110]$. The main difficulty in top-down methods is to overcome the van der Waals forces that hold the graphene layers together in graphite, preventing reagglomeration and avoiding damages in the honeycomb carbon structure [111,112]. Some examples of such methods are micromechanical exfoliation, direct sonication, electrochemical exfoliation, and superacid dissolution. Bottom-up methods include chemical vapor deposition (CVD), arc discharge, and epitaxial growth on silicon carbide [104].

The structure of CNT can be conceptualized by wrapping graphene into a cylinder. Typically, CNT are classified as either single-walled carbon nanotubes (SWCNT) or multi-walled carbon nanotubes (MWCNT). SWCNT exhibit better electrical properties, while MWCNT display better chemical resistance [113].

CNT can be produced using different methods, which mainly involve gas phase processes [114,115], like CVD, arc discharge, and laser ablation [116]. The most commonly used and efficient methods are the ones involving CVD, in which a carbon containing source (e.g., methane, acetylene, ethylene) reacts with a metal catalyst particle (e.g., iron, cobalt, nickel) which act as growth nuclei for CNT, at temperatures above $600{ }^{\circ} \mathrm{C}$. There are several substrate materials for catalyst particles, as graphite, quartz, silicon, silicon carbide, amongst others. It is pertinent to mention that for graphene production by this technique, no catalyst particles are used, being the substrate itself a catalytic metal, often copper for monolayer or nickel for few layer graphene. Generally, CVD has the advantages of allowing mild and controllable synthesis in large scale [117-120].

CNT are strong, flexible, electrically conductive, and can be functionalized [121]. Potential applications of CNT have been reported such as in composite materials [122], electrochemical devices [123], hydrogen storage [124], field emission devices [125], nanometer-sized electronic devices, sensors and probes [126]. Determining the toxicity of CNT has been one of the most pressing questions in nanotechnology [127]. There is still some controversy on this subject, thus continued research is needed to assure that these materials are safe for biomedical applications $[128,129]$. Parameters such as structure, size distribution, surface area, surface chemistry, surface charge and agglomeration state, as well as the sample purity, have considerable impact on CNT properties [121].

In the research works reported in this review, $\mathrm{CBN}$ are both commercial products or lab-made by the authors. Most commercial CNT are produced by CVD, with suppliers often making available information about material dimensions and sometimes type of CVD used. On the other hand, researchers usually produce GBM from graphitic precursors, using top-down methods involving chemical oxidation and exfoliation, namely the Staudenmaier and modified Hummers methods (Figure 1). Commercial GBM are also used, with suppliers giving information about dimensions, and sometimes production methods. These involve direct exfoliation in a liquid, with or without the use of a surfactant, or in the solid state by edge functionalization, or by first inserting a chemical species between 
the graphene layers in graphite to weaken their interaction, followed by expansion/exfoliation [130]. Commercial products offer insured reproducibility and widespread availability. Moreover, with the optimization of the production processes, the costs of GBM are coming closer to its precursor, graphite [11].
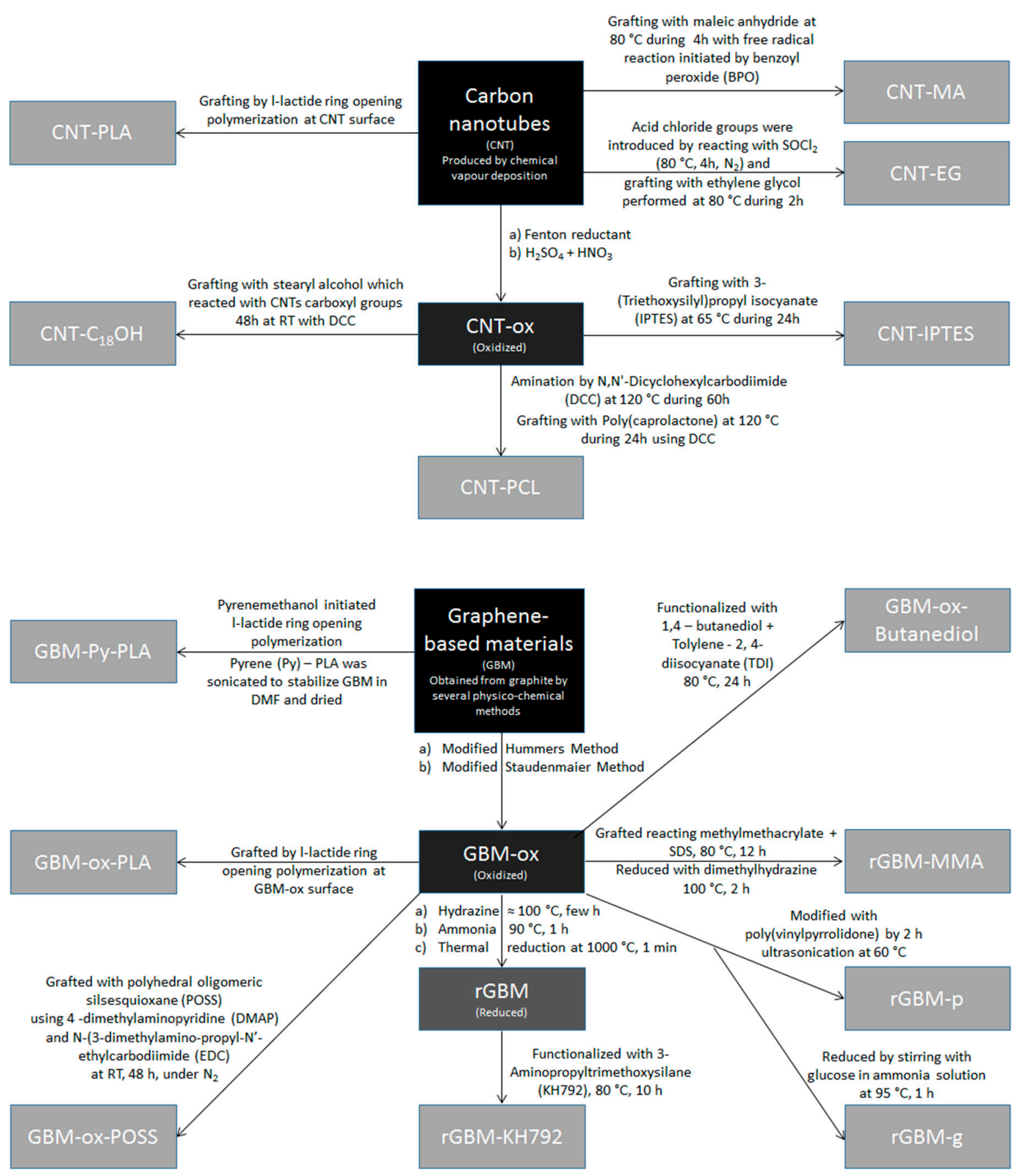

Figure 1. Scheme showing the different types of modifications performed on carbon-based nanomaterials (CBN) prior to incorporation in poly(lactic acid) (PLA). 
CBN have been extensively used in polymer composites. In order to take advantage of their large surface area maximizing its effectiveness as filler, dispersion must be efficient, so as to maximize the amount of deagglomerated primary units. Functionalization is often used to improve compatibility with the polymer matrix. However, this can disrupt the $\mathrm{sp}^{2}$ hybridization of CBN carbon structure and subsequently hinder their properties [131]. Some examples of CBN modifications used on the research works reported in this review are compiled in Figure 1. Some of these involve simple chemical oxidation, prior to surface modification with isocyanates, polymers (ethylene glycol, poly(caprolactone), methyl methacrylate, poly(vinyl pyrrolidone), and PLA), polyols or silanes. The impact of these on the composite properties is discussed in Section 5.

\section{Production of PLA/CBN Composites}

Three methods are most frequently used to obtain a dispersion of CBN into a polymer matrix: solution mixing, melt blending, and in situ polymerization [22,104]. Mechanical milling, also called ball milling, has been gaining recognition as an alternative technique with specific advantages, but it has not yet been reported for PLA/CBM composites. High impact milling is performed at room temperature on dry powders, prior to melt processing. Its effectiveness and benefits in relation to other methods have been shown for different polymer/filler systems [132].

\subsection{Solution Mixing}

Solution mixing is a simple procedure, requiring no special equipment, and allowing for straightforward scale-up. This method typically consists of three steps: (i) dispersion of the nanomaterial in a suitable solvent using sonication or mechanical stirring; (ii) dissolution of the polymer in the previous dispersion, under appropriate stirring; and (iii) removal of the solvent by distillation or lyophilization. Often the dispersion is cast into a flat mold, and then the solvent is evaporated. Flat composite slabs are therefore obtained. For this reason, the procedure is often called "solvent casting". As an alternative, the dispersion may be cast onto a low surface energy material (e.g., PTFE coated surface) using a blade applicator (doctor blading). After solvent evaporation, thin composite films are obtained. The viscosity of the dispersion needs to be adjusted for this procedure, which can be done by changing the concentration of polymer [133]. If production of fibers is desired, the third step can be replaced by electrospinning. This technique allows obtaining fibers that are much smaller in diameter (ranging from micrometers to nanometers) than those produced by conventional techniques. The basis of electrospinning is to charge the polymer solution in the spinneret tip with a high voltage, so that the electrostatic repulsion overcomes the surface tension of the solution, causing its ejection. The solvent vaporizes while the jet is in the air, producing a continuous fiber which deposits on the ground collector [27].

Complete solvent removal is a critical issue when using solution mixing to prepare composites, since toxicity concerns may arise when organic solvents are used. In addition, presence of residual solvent induces plasticization of the polymer matrix, which may alter significantly its mechanical properties [134-136].

PLA is soluble in organic solvents such as chlorinated solvents, benzene, tetrahydrofuran (THF), dimethyl formamide (DMF) and dioxane, but insoluble in ethanol, methanol, and aliphatic hydrocarbons. CBN are hydrophobic, therefore cannot be easily dispersed in polar solvents. However, they can be oxidized or modified with hydrophilic groups in order to allow dispersion in such solvents. Solubility limitations can also be overcome to a certain point by using ultrasonication to produce short-time metastable dispersions of CBN in organic solvents, which can then be mixed with polymer solutions [137].

Chloroform is the most used solvent to prepare PLA/CNT composites [138-143]. Despite, some authors obtain good results with THF [88,144], and dichloromethane [145,146]. McCullen et al. [147] conclude that a combination of chloroform and DMF is beneficial. Sometimes the introduction of new functional groups may originate incompatibility with the polymer matrix. To elude this problem, 
improvement of CNT dispersion by surfactant addition (e.g., polyoxyethylene 8 lauryl, dodecyl octaethylene) may be used, which allows preserving the chemical structure of the nanofiller [148]. GBM have been often incorporated in PLA by solution mixing using chloroform [135,149-151] or DMF [152-157] as solvents. Agglomeration of CBN may take place during solvent evaporation. Composite formation by electrospinning allows minimizing this problem, but leads to formation of fibers and not films $[27,147,158]$.

\subsection{Melt Blending}

Melt blending is an economically attractive, environmentally friendly and highly scalable method for preparing nanocomposites. This strategy involves direct addition of the nanomaterial into the molten polymer, allowing optimization of the state of dispersion by adjusting operating parameters such as mixing speed, time and temperature. Due to the absence of solvent, the only compatibility issue is placed in terms of the nanofiller towards the polymer matrix $[27,48]$. The drawbacks of this procedure are the low bulk density of $\mathrm{CBN}$, that makes the feeding of the melt-mixer a troublesome task and the lower degree of dispersion that is usually attained when compared to solvent mixing [137,159].

Most published research works use a lab-scale melt mixer to melt PLA and mix it with the nanofillers. Typical processing conditions correspond to temperatures between $160{ }^{\circ} \mathrm{C}$ and $180{ }^{\circ} \mathrm{C}$ [160-166], mixing times of 5 to $10 \mathrm{~min}$ [160-162,164,165,167], and rotation speeds between 50 and 100 rpm [160-164,166-169]. After mixing, the composite materials are almost always molded into flat sheets with controlled thickness in a hot press, however, other methods are also used (e.g., injection molding and piston spinning). Typically, the pressing is performed between $160{ }^{\circ} \mathrm{C}$ and $190{ }^{\circ} \mathrm{C}$ for 2 to $5 \mathrm{~min}$, under 110 to $150 \mathrm{Kgfcm}^{-2}$ pressure [160,165-170].

In addition to melt blending not being as effective as the solution mixing method or in situ polymerization in terms of the ability to achieve good filler dispersion, damage to the nanofillers or polymer may occur under severe conditions. Some studies have shown that processing conditions can have an impact on the molecular weight of PLA [171]. This can be mainly attributed to the presence of impurities such as acidic species, peroxide groups, metallic ions or other residual products that can increase the degradation of PLA during melt mixing [172].

\subsection{In Situ Polymerization}

In situ polymerization for production of polymer composites generally involves mixing the filler in neat monomer, or a solution of monomer, in the presence of catalysts and under proper reaction conditions [173]. The polymer chains grow on the filler surface, being covalently bonded. In situ polymerization generally results in more homogeneous particle dispersion than melt blending [174]. Use of this approach for polymerizing lactide in the presence of CNT has been reviewed by Brzeziński and Biela [175]. Contrary to CNT, that usually are post-treated, GBM already present some chemical groups that can be used in further functionalization, such as grafting polymer chains via atom transfer radical polymerization. Examples of in situ polymerization on GBM include polymers such as polyaniline (PANI), polyurethane (PU), polystyrene (PS), poly(methyl methacrylate) (PMMA) and polydimethylsiloxane (PDMS) [24].

Concerning the particular case of PLA/CBN, only a few examples of in situ polymerization can be found in the literature. Ring opening polymerization of L-lactide in presence of GBM has been reported by Yang et al. [176] and Promoda et al. [177]. Carboxyl-functionalized CNT have been grafted with PLA by Li and co-workers [178].

The above-mentioned composite production methods can be used both with GBM and CNT, and are congregated in Figure 2. 


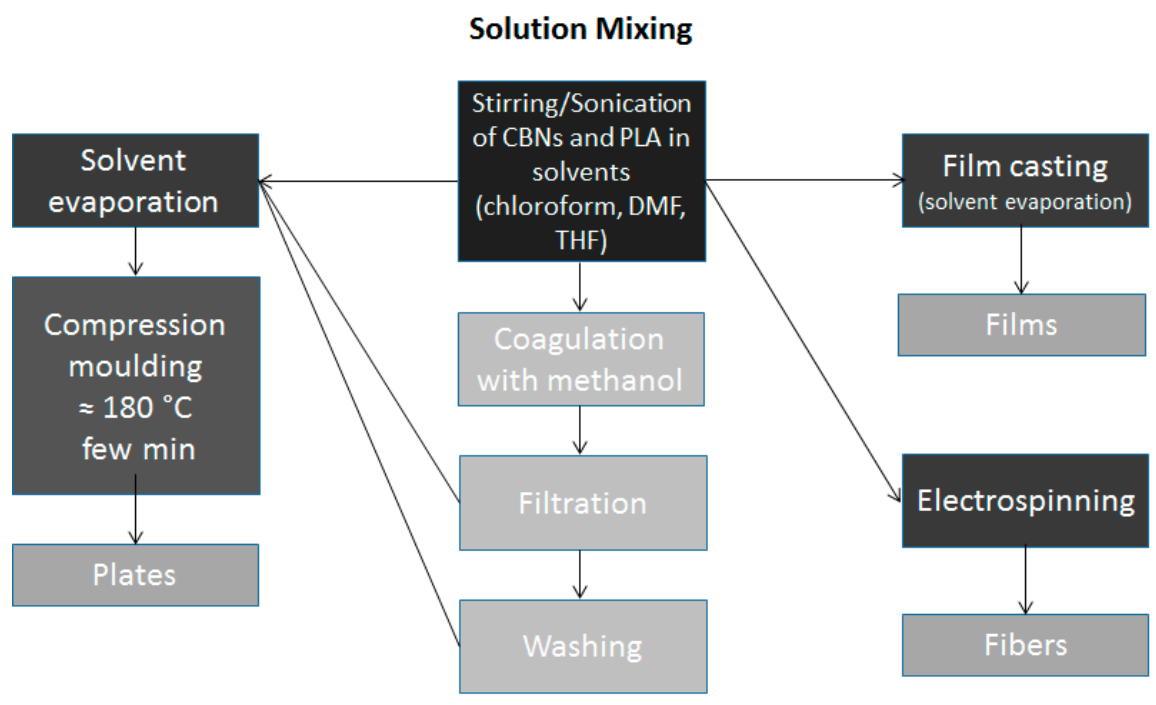

Melt blending

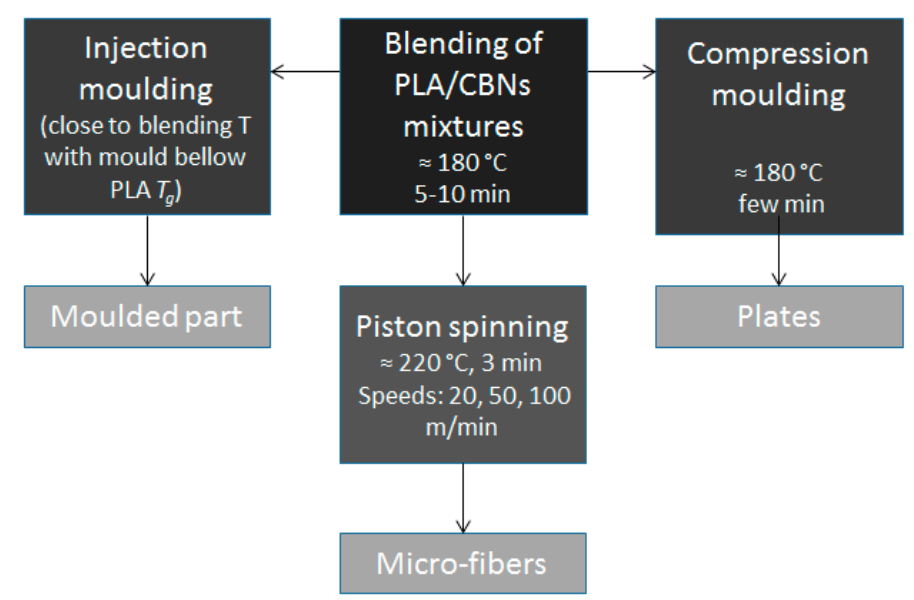

\section{In-situ polymerization}



Figure 2. Scheme showing the different production methods of PLA/CBN composites.

\section{Properties of PLA/CBN Composites}

Numerous researchers have studied the properties of PLA combined with other materials, in order to tune key properties regarding specific applications [48]. The current review is focused on the effect of incorporating two carbon-based nanomaterials, CNT and GBM, in PLA. CNT are known for two decades and have well established large-scale production methods. GBM, which have been raising a growing interest from the scientific community, are cheaper and, in principle, comparable in properties to CNT [177].

\subsection{Mechanical Properties}

Physico-chemical interactions between fillers and polymer phase contribute to load transfer and distribution along the CBN network. Table 1 shows that solution mixing is the most commonly reported method for incorporation of CBN in PLA. The most frequently used solvents are chloroform, DMF and 
THF. The filler concentrations most often tested are between 0.1-2 wt \%. Maximum improvements in Young's modulus $(E)$, storage modulus $\left(E^{\prime}\right)$, and tensile strength $\left(\sigma_{\max }\right)$ are found for concentrations between $0.25-5 \mathrm{wt} \%$ for CNT, and between $0.1-1 \mathrm{wt} \%$ for GBM. The larger improvement in $E$, relative to unfilled PLA, is of $372 \%$, for $0.25 \mathrm{wt} \%$ MWCNT sonicated in a PLA/chloroform dispersion, followed by compression molding of the dried mixture [138]. For GBM, the best performance is an increase of $156 \%$ with incorporation of $0.4 \mathrm{wt} \%$ GNP-M, also by sonication, but followed by film casting using doctor blading. In this study, comparison is made with GO, which yields a maximum $E$ increase at $0.3 \mathrm{wt} \%$ loading. Figure 3 presents microscopy images demonstrating good dispersion of the fillers in the PLA matrix [135].

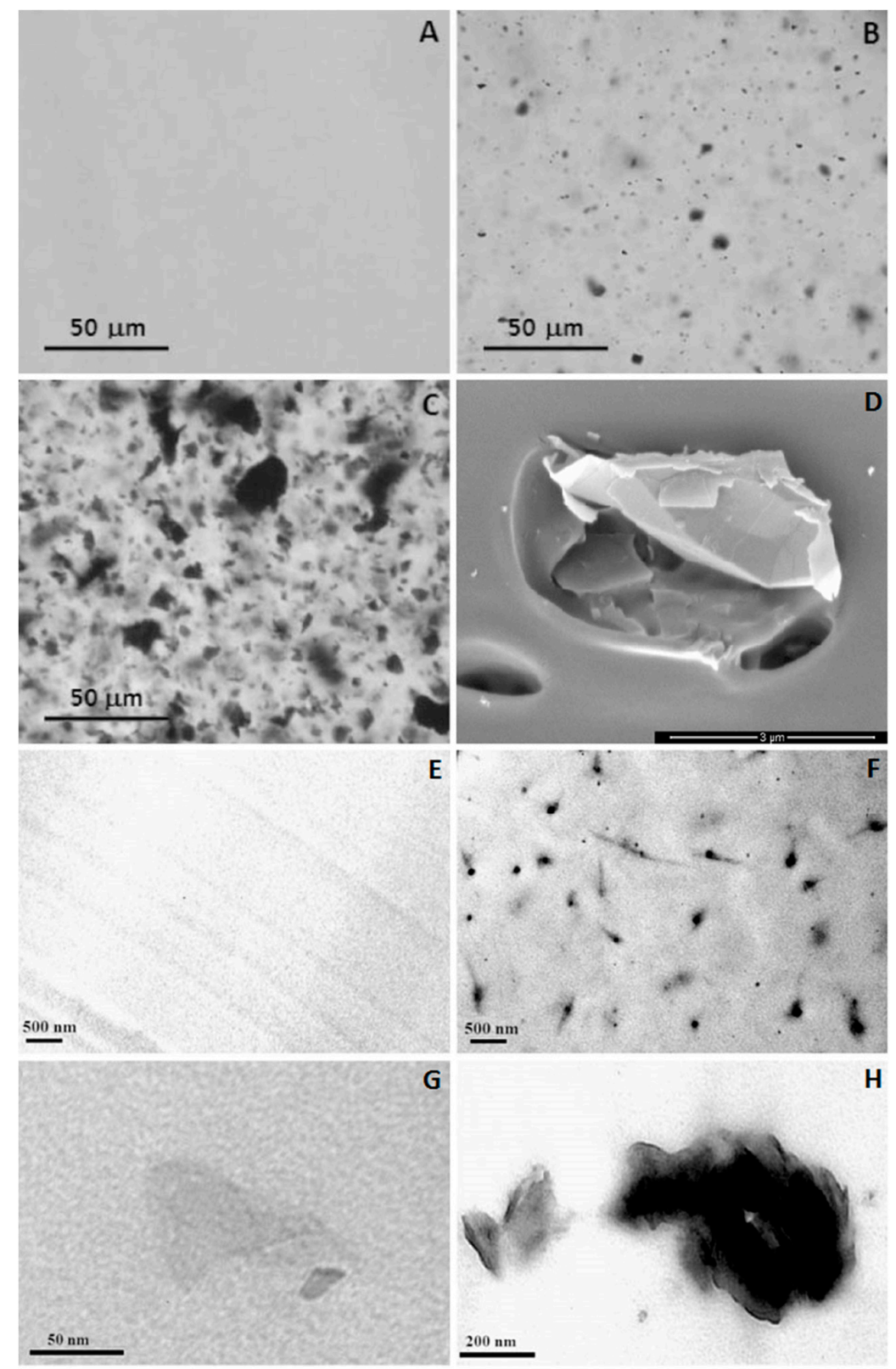

Figure 3. Microscopy images of PLA, PLA/GNP and GO $0.4 \mathrm{wt} \%$ films produced by solution mixing followed by film casting using doctor blading, displaying good filler dispersion and interaction with polymer matrix. Optical microscopy images of PLA (A); PLA/GO (B); and PLA/GNP (C); Scanning electron microscopy image of PLA/GNP (D); Transmission electron microscopy images of PLA (E) and PLA/GO (F-H) [179]. 
The maximum increase on $E^{\prime}$ is of $1500 \%$, achieved with incorporation of $0.5 \mathrm{wt} \% \mathrm{rGO}-\mathrm{KH} 792$ in PLLA, by simple stirring, casting on PTFE mold, and vacuum drying the resultant films at $120^{\circ} \mathrm{C}$ for $48 \mathrm{~h}$ [157]. However, this increase only occurs around PLA transition temperature $\left(60-65^{\circ} \mathrm{C}\right)$. At ambient temperature, the best result is an increase of $67 \%$ with incorporation of $3 \mathrm{wt} \%$ A-SWCNT-Si (acid treated and grafted with 3-isocyanatoporpyl triethoxysilane) in PLA by sonication, followed by drying and compression molding at $190{ }^{\circ} \mathrm{C}$ [144]. The maximum increase in $\sigma_{\max }$ is of $129 \mathrm{wt} \%$, obtained with incorporation of $0.4 \mathrm{wt} \%$ GNP-M in PLA by sonication and film casting by doctor blading [135]. For CNT the best result is an increase of $47 \%$ obtained with MWCNT grafted with PLA, and then incorporated at a loading of $1 \mathrm{wt} \%$ in PLA by sonication in chloroform, separation, drying and compression molding at $180^{\circ} \mathrm{C}$ [141]. When considering CNT without modification, the best result reported is an increase of $9 \%$ for $1.2 \mathrm{wt} \%$ MWCNT incorporated in PLA by solution mixing, followed by drying and compression molding at $180^{\circ} \mathrm{C}$ with a pressure of $1000 \mathrm{Kg}$ [142].

Melt-blending is less frequently reported than solution mixing for production of PLA/CBN composites, probably due to the lower availability of the necessary equipment. Results show that it tends to be not as effective in improvement of mechanical properties, as solution mixing. The best performance in terms of $E(\uparrow 88 \%)$ and $E^{\prime}(\uparrow 76 \%)$ is reported by Lin et al. [160] for an incorporation of $3 \mathrm{wt} \%$ MWCNT grafted with stearyl alcohol (MWCNT- $\left.\mathrm{C}_{18} \mathrm{OH}\right)$ in PLA by melt blending $\left(180^{\circ} \mathrm{C}\right.$, $5 \mathrm{~min}, 50 \mathrm{rpm})$, using $\mathrm{Ti}(\mathrm{OBu})_{4}$ for transesterification, followed by compression molding at the same temperature. When PLA is not transesterified, $E$ and $E^{\prime}$ increases were of $74 \%$ and $44 \%$, respectively. The maximum increase in $\sigma_{\max }(40 \%)$ is obtained incorporating $0.08 \mathrm{wt} \% \mathrm{rGO}$ using a twin-screw mixer $\left(175{ }^{\circ} \mathrm{C}, 8 \mathrm{~min}, 60 \mathrm{rpm}\right)$, followed by compression molding at $180{ }^{\circ} \mathrm{C}$ [168]. The incorporation by melt blending $\left(180^{\circ} \mathrm{C}, 20 \mathrm{~min}, 50 \mathrm{rpm}\right)$ of $0.25 \mathrm{wt} \%$ GNP-M 5 and C in PLA followed by compression molding at $190^{\circ} \mathrm{C}$, prevented its mechanical properties decay after 6 months degradation in phosphate-buffered saline at $37^{\circ} \mathrm{C}[180]$.

In situ polymerization is the least used technique. It has been reported by Pramoda et al. [177], who performed PLA ring-opening polymerization in presence of $1 \mathrm{wt} \%$ of GO functionalized with butanediol and GO modified with POSS silsesquioxane. In the first case, improvements of $1 \%$ and $14 \%$ in $E$ and hardness are obtained, respectively. In the second, the performance is increased by $33 \%$ and $45 \%$, in the same order.

Comparing the results for CNT and GBM, we can conclude that both can effectively improve PLA mechanical properties, whether by solution mixing and melt blending. However, use of GBM usually implies lower amounts of GBM than of CNT. Several chemical modifications have been tried to improve compatibility with the polymer matrix, with ineffective results is some cases. Functionalization with carboxyl groups is the most common and effective procedure to improve CNT compatibility with PLA matrix [146]. On the other hand, no relation has been observed between CBN morphological properties (size, length, and diameter) and the mechanical performance of the composites. 
Table 1. Mechanical properties of PLA/CBN composites in comparison with non-modified PLA. Production methods and CBN characteristics.

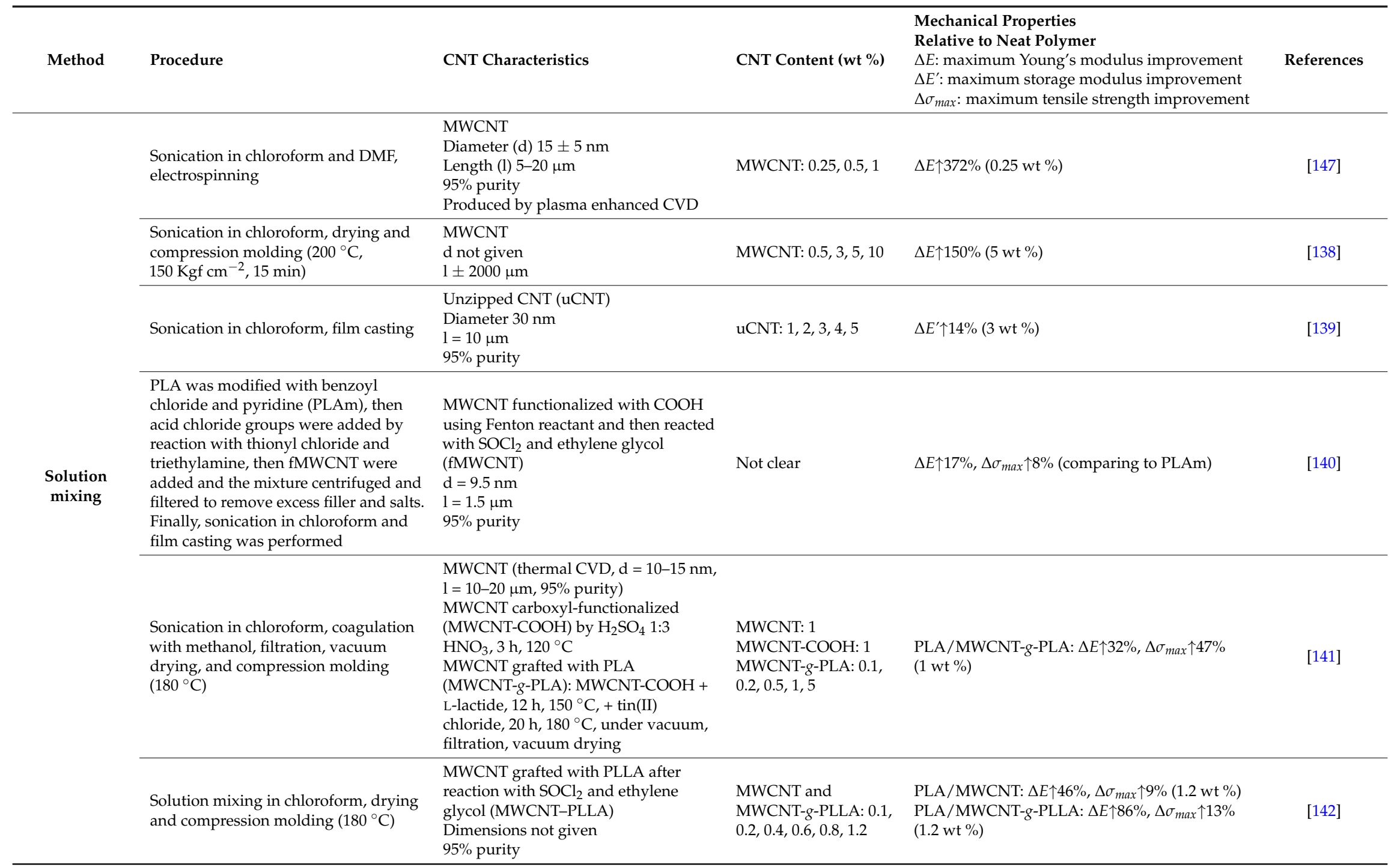


Table 1. Cont.

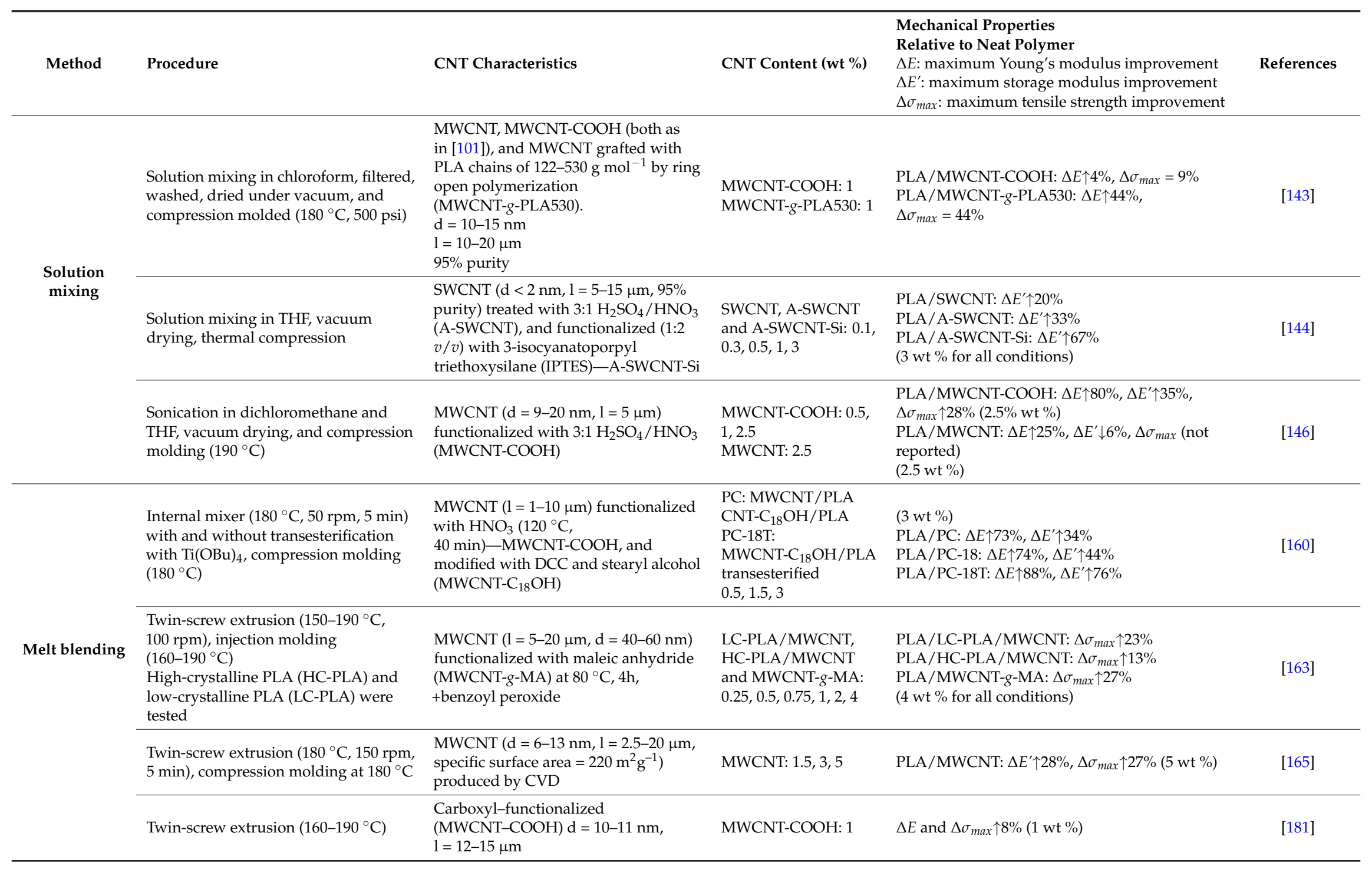


Table 1. Cont

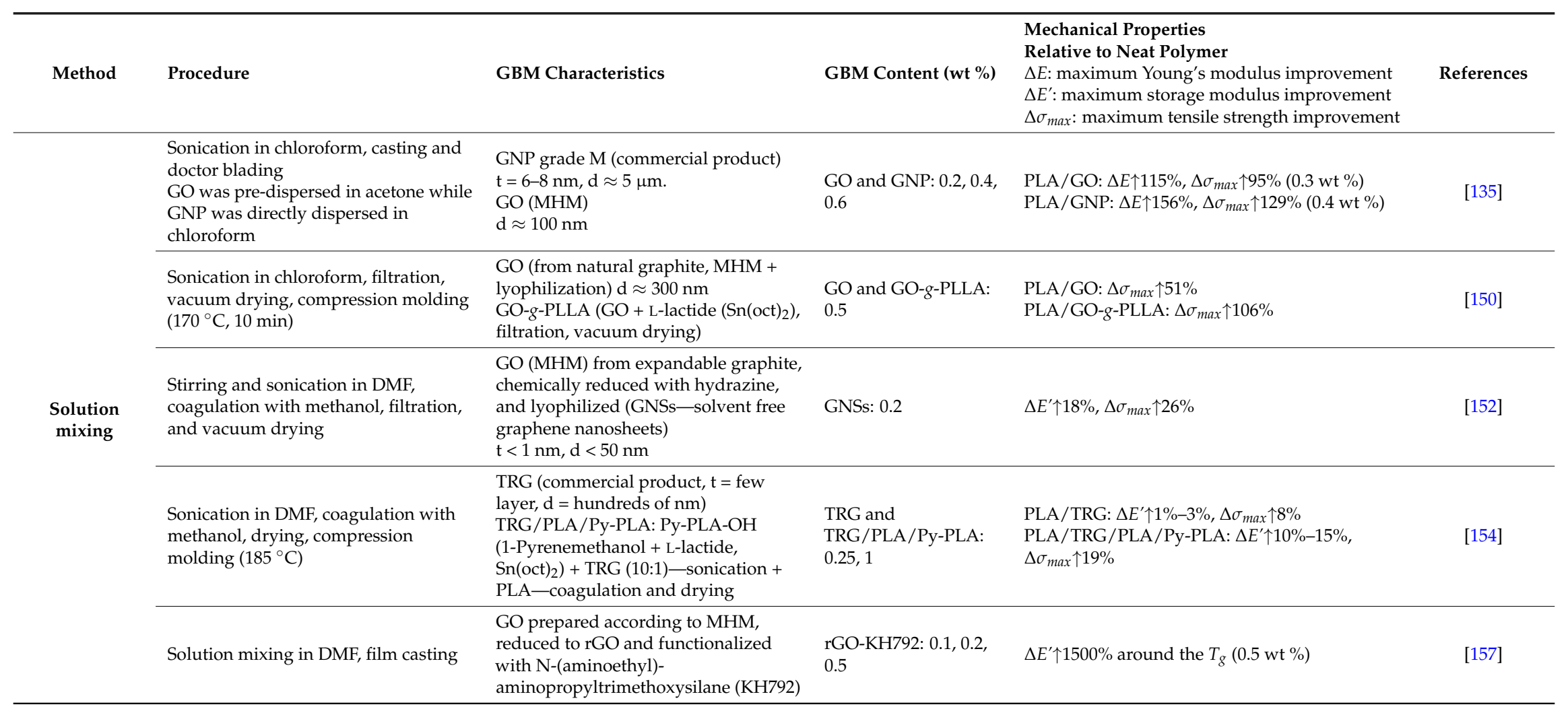


Table 1. Cont.

\begin{tabular}{|c|c|c|c|c|c|}
\hline Method & Procedure & GBM Characteristics & GBM Content (wt \%) & $\begin{array}{l}\text { Mechanical Properties } \\
\text { Relative to Neat Polymer } \\
\Delta E: \text { maximum Young's modulus improvement } \\
\Delta E^{\prime}: \text { maximum storage modulus improvement } \\
\Delta \sigma_{\text {max }} \text { : maximum tensile strength improvement }\end{array}$ & References \\
\hline \multirow{4}{*}{ Melt blending } & $\begin{array}{l}\text { Twin-screw mixer }\left(175^{\circ} \mathrm{C}, 60 \mathrm{rpm},\right. \\
8 \mathrm{~min}) \text {, compression molding at } 180^{\circ} \mathrm{C}\end{array}$ & $\begin{array}{l}\text { GO prepared by MHM and reduced } \\
\text { with hydrazine and ammonia }(\mathrm{rGO}) \mathrm{t}= \\
0.4-0.6 \mathrm{~nm}, \mathrm{~d}=0.1-0.5 \mu \mathrm{m}\end{array}$ & $\begin{array}{l}\text { rGO: } 0.02,0.04,0.08, \\
0.2,0.5,1,2\end{array}$ & $\begin{array}{l}\Delta E^{\prime} \uparrow 27 \%, \Delta \sigma_{\max } \uparrow 40 \%(0.08 \mathrm{wt} \%) \\
\Delta E^{\prime} \uparrow 54 \%, \Delta \sigma_{\max } \downarrow 40 \%(2 \mathrm{wt} \%)\end{array}$ & [168] \\
\hline & $\begin{array}{l}\text { Internal mixer }\left(160^{\circ} \mathrm{C}, 25 \mathrm{rpm}, 10 \mathrm{~min}\right) \\
\text { compression molding }\left(160^{\circ} \mathrm{C}, 10 \mathrm{~min}\right) \\
\text { (Polymer was PLA/PEG 9:1 blend) }\end{array}$ & $\begin{array}{l}\text { GNP grade M15 (commercial product) } \\
\mathrm{t}=6-8 \mathrm{~nm}, \mathrm{~d} \approx 15 \mu \mathrm{m}\end{array}$ & $\begin{array}{l}\text { GNP-M15: 0.1, 0.3, 0.5, } \\
0.7,1\end{array}$ & $\begin{array}{l}\Delta E^{\prime} \uparrow 84 \text { and } 70 \%, \Delta \sigma_{\max } \uparrow 20 \text { and } 33 \% \\
\text { (0.1 and } 0.3 \mathrm{wt} \% \text { ) } \\
\text { (relative to pristine PLA/PEG blend) }\end{array}$ & [167] \\
\hline & $\begin{array}{l}\text { Internal mixer }\left(180^{\circ} \mathrm{C}, 80 \mathrm{rpm}, 10 \mathrm{~min}\right) \\
\text { Compression molding }\left(180^{\circ} \mathrm{C}\right)\end{array}$ & $\begin{array}{l}\mathrm{GO}(\mathrm{MHM})+\mathrm{SDS} \text {, ultrasounds, } \\
\text { stirring } 12 \mathrm{~h}, 25^{\circ} \mathrm{C} \\
\text { Methylmethacrylate (MMA), stirring } \\
12 \mathrm{~h}+\text { ammonium persulfate (APS) } \\
12 \mathrm{~h}, 80^{\circ} \mathrm{C}+\text { reduction with dimethyl } \\
\text { hydrazine, } 100^{\circ} \mathrm{C}, 2 \mathrm{~h} \\
\text { (PFG-polymer-functionalized } \\
\text { graphene nanoparticles) } \\
\mathrm{t}=2.4 \mathrm{~nm}\end{array}$ & PFG: $1,2,3,4,5$ & $\Delta E \uparrow 80 \%, \Delta \sigma_{\max } \uparrow 10 \%$ (5 wt \%) & [164] \\
\hline & $\begin{array}{l}\text { Internal mixer }\left(180^{\circ} \mathrm{C}, 50 \mathrm{rpm}, 20 \mathrm{~min}\right) \\
\text { Compression molding }\left(190^{\circ} \mathrm{C}, 2 \mathrm{~min} \text {, }\right. \\
\left.150 \mathrm{Kg} \mathrm{cm}^{-2}\right)\end{array}$ & $\begin{array}{l}\text { GNP grade M5 } \\
(\mathrm{t}=6-8 \mathrm{~nm}, \mathrm{~d} \approx 5 \mu \mathrm{m}) \text { and } \mathrm{C}(\mathrm{t}=\text { up to } \\
2 \text { single layers, } \mathrm{d}<2 \mu \mathrm{m}) \\
\text { (commercial products) }\end{array}$ & $\begin{array}{l}\text { GNP-M5 and C: } 0.1, \\
0.25,0.5\end{array}$ & $\begin{array}{l}\text { PLA/GNP-M5: } \Delta E \uparrow 14 \%, \Delta \sigma_{\max } \uparrow 6 \%(0.25 \mathrm{wt} \%) \\
\text { PLA/GNP-C: } \Delta E \uparrow 14 \%, \Delta \sigma_{\max } \uparrow 20 \%(0.25 \mathrm{wt} \%) \\
\text { The incorporation of both fillers prevented } \\
\text { mechanical properties decay after } 6 \text { months } \\
\text { degradation }\end{array}$ & {$[180,182]$} \\
\hline $\begin{array}{c}\text { In situ } \\
\text { polymerization }\end{array}$ & $\begin{array}{l}\text { Sonication of L-lactide + filler in } \\
\text { toluene, addition of } \\
\text { Tin(II)-2-ethylhexanoate under } \mathrm{N}_{2} \text {, } \\
\text { stirring at } 110^{\circ} \mathrm{C}, 3 \text { days }\end{array}$ & $\begin{array}{l}\text { Expanded graphite (MHM) to GO } \\
\text { GO-functionalized: GO + TDI + } \\
\text { 1,4-butanediol, } 80^{\circ} \mathrm{C}, 24 \mathrm{~h} \\
\text { GO-g-POSS: GO + POSS-polyhedral } \\
\text { oligomeric silsesquioxane + } \\
\text { DMAP-4-(dimethylaminopyridine) + } \\
\text { EDC- } \mathrm{N} \text {-(3-dimethylamino-propyl- } \mathrm{N}^{\prime} \text { - } \\
\text { ethylcarbodiimide), } 2 \text { days, room } \\
\text { temperature, } \mathrm{N}_{2} \\
\text { (dimensions not given) }\end{array}$ & $\begin{array}{l}\text { GO-functionalized, } \\
\text { GO-g-POSS, } \\
\text { GO+POSS (physical } \\
\text { mixture): } 1\end{array}$ & $\begin{array}{l}\text { PLA/GO-functionalized: } \\
\Delta E^{\prime} \uparrow 1 \%, \text { Hardness } \uparrow 14 \% \\
\text { PLA/GO-g-POSS: } \\
\Delta E^{\prime} \uparrow 33 \% \text {, Hardness } \uparrow 45 \% \\
\text { PLA/GO + POSS: } \\
\Delta E^{\prime} \uparrow 29 \% \text {, Hardness } \uparrow 36 \%\end{array}$ & [177] \\
\hline
\end{tabular}




\subsection{Electrical Properties}

Neat PLA is electrically insulating with a low electrical conductivity $\left(\sigma \approx 1 \times 10^{-16} \mathrm{~S} \mathrm{~m}^{-1}\right)$, and high sheet resistance $\left(\rho_{\square} \approx 5 \times 10^{12} \Omega \mathrm{sq}^{-1}\right)[144,160]$. Since CNT and reduced forms of GBM present high electrical conductivity, they can be incorporated in PLA to improve its conductivity. This sort of composites have potential to be used as electrical stimulating implants, since PLA is used as a biodegradable matrix in orthopedic material. Other advantages of increasing PLA conductivity are the possibility of using it as antistatic coating/material or for electromagnetic shielding [104]. The minimum amount of filler required to form a conductive network within the polymer is called percolation threshold, and should be as low as possible in order to keep processing simple (relatively low viscosity of the melt) and low costs. Table 2 shows that, once again, the most used method to incorporate CBN on PLA for electrical properties evaluation is solution mixing. The amount of fillers ranges from 0.01 to $10 \mathrm{wt} \%$. The best result, considering electrical conductivity $(\sigma)$ with CNT is $3.5 \times 10^{-3} \mathrm{~S} \mathrm{~m}^{-1}$, obtained incorporating $10 \mathrm{wt} \%$ MWCNT in PLA by sonication in chloroform, followed by drying and compression molding at $200{ }^{\circ} \mathrm{C}$ during $15 \mathrm{~min}$ [138]. Results are also often presented in terms of sheet resistance $\left(\rho_{\square}\right)$, being the lowest value reported by Shao et al. [183], of $1 \times 10^{2} \Omega \mathrm{sq}^{-1}$ achieved incorporating $5 \mathrm{wt} \%$ MWCNT previously oxidized (treated with $\mathrm{HCl}$ and $\mathrm{HNO}_{3}$ ) in PLA by solution mixing, followed by electrospinning of aligned nanofibers $(\mathrm{d} \approx 250 \mathrm{~nm})$. The alignment of the fibers slightly improved sheet resistance, comparing with random meshes. Interestingly, Yoon et al. [143] observe a considerable sheet resistance of $1 \times 10^{5} \Omega \mathrm{sq}^{-1}$, with incorporation of $1 \mathrm{wt} \%$ MWCNT-COOH, also oxidized by treatment with strong acids $\left(\mathrm{H}_{2} \mathrm{SO}_{4}\right.$ and $\mathrm{HNO}_{3}$ ). For GBM, the maximum conductivity reported is $2.2 \mathrm{~S} \mathrm{~m}^{-1}$, higher than for CNT, obtained incorporating $1.25 \mathrm{wt} \% \mathrm{rGO}-\mathrm{g}$ (reduced with ammonia) in PLA by sonication in DMF. Interestingly, the solvent used for dispersion of CNT in PLA is always chloroform and for GBM is always DMF.

Melt-blending is the second most used approach to disperse CBN in PLA in order to improve its electrical properties, being most often performed by twin-screw extrusion, followed by compression molding. The highest $\sigma$ considering CNT is $50 \mathrm{~S} \mathrm{~m}^{-1}$, which is reported by Pötschke et al. [184]. These authors prepare MWCNT mixtures by twin-screw extrusion, followed by piston spinning at different speeds. They conclude that non-spun mixtures with $5 \mathrm{wt} \%$ MWCNT in PLA present the same conductivity as $3 \mathrm{wt} \%$ mixtures after piston spinning at a speed of $20 \mathrm{~m} \mathrm{~min}^{-1}$. Microscopy images in Figure 4 allow to observe good MWCNT dispersion and orientation due to spinning process.

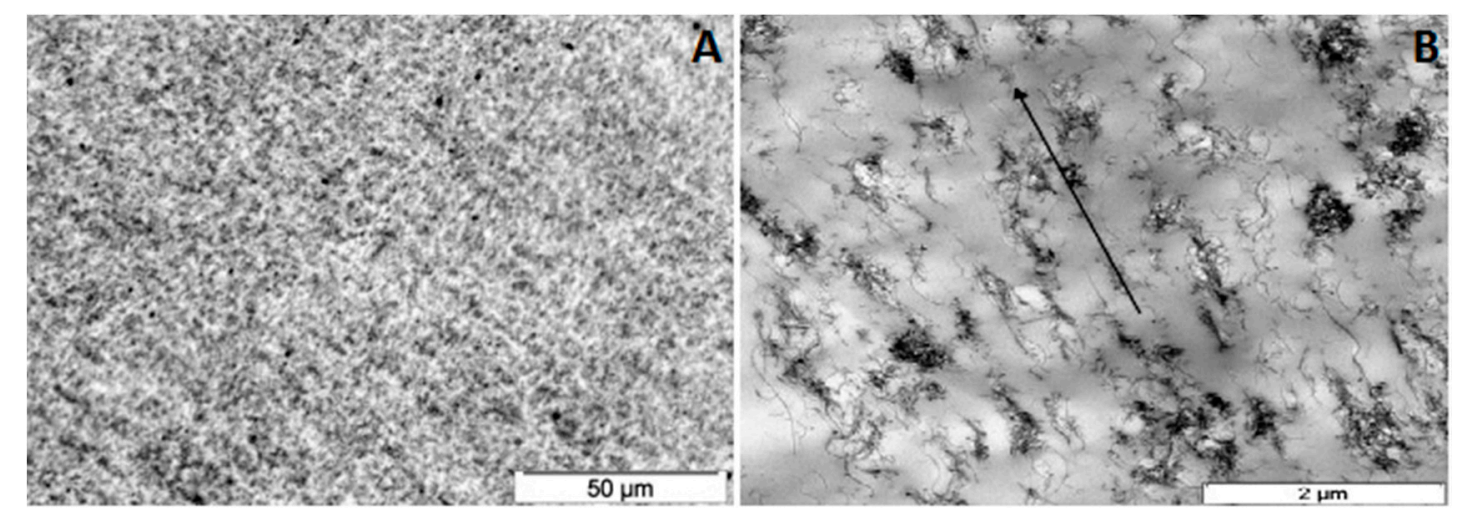

Figure 4. Optical microscopy image of a PLA/MWCNT 3 wt \% mixture produced by twin-screw extrusion (A) -illustrating the high degree of macroscopic filler dispersion. Transmission electron microscopy image of a PLA/MWCNT $3 \mathrm{wt} \%$ mixture produced by twin-screw extrusion, followed by piston spinning; (B) - arrow indicates that fillers are strongly oriented in fiber direction due to the spinning process [185]. 
Table 2. Electrical properties of PLA/CBN composites in comparison with non-modified PLA. Production methods and CBN characteristics.

\begin{tabular}{|c|c|c|c|c|c|}
\hline Method & Procedure & CNT Characteristics & CNT Content (wt \%) & $\begin{array}{l}\text { Electrical Properties } \\
\sigma: \text { electrical conductivity } \\
\rho_{\square} \text { : sheet resistance } \\
\left(\text { PLA } \sigma \approx 1 \times 10^{-16} \mathrm{~S} \mathrm{~m}^{-1},\right. \\
\left.\rho_{\square} \approx 5 \times 10^{12} \Omega \mathrm{sq}^{-1}\right)[106,122]\end{array}$ & References \\
\hline \multirow{5}{*}{$\begin{array}{l}\text { Solution } \\
\text { mixing }\end{array}$} & $\begin{array}{l}\text { Sonication in chloroform, } \\
\text { coagulation with methanol, } \\
\text { filtration, vacuum drying, and } \\
\text { compression molding }\left(180^{\circ} \mathrm{C}\right)\end{array}$ & $\begin{array}{l}\text { MWCNT (thermal CVD, } \mathrm{d}=10-15 \mathrm{~nm}, \\
\mathrm{l}=10-20 \mu \mathrm{m}, 95 \% \text { purity) } \\
\text { MWCNT carboxyl-functionalized } \\
\left(\mathrm{MWCNT}-\mathrm{COOH} \text { ) by } \mathrm{H}_{2} \mathrm{SO}_{4} 1: 3 \mathrm{HNO}_{3}, 3 \mathrm{~h},\right. \\
120^{\circ} \mathrm{C} \\
\text { MWCNT grafted with PLA (MWCNT-g-PLA): } \\
\text { MWCNT-COOH + L-lactide, } 12 \mathrm{~h}, 150{ }^{\circ} \mathrm{C},+ \\
\text { tin(II) chloride, } 20 \mathrm{~h}, 180^{\circ} \mathrm{C}, \text { under vacuum, } \\
\text { filtration, vacuum drying }\end{array}$ & $\begin{array}{l}\text { MWCNT: } 1 \\
\text { MWCNT-COOH: } 1 \\
\text { MWCNT- } g \text {-PLA:0.1, } 0.2 \\
0.5,1,5\end{array}$ & $\begin{array}{l}\text { PLA/MWCNT: } \rho_{\square}=1 \times 10^{12} \Omega \mathrm{sq}^{-1} \text { (for } 0.1 \\
\text { and } 0.2 \mathrm{wt} \% \text { is similar to PLA), } 1 \times 10^{5} \text { and } \\
1 \times 10^{4} \Omega \mathrm{sq}^{-1}(0.5 \mathrm{wt} \% \text {, and } 1-5 \mathrm{wt} \%) \\
\text { PLA/MWCNT-g-PLA: } \rho_{\square}=1 \times 10^{12} \Omega \mathrm{sq}^{-1} \\
(0.1-5 \mathrm{wt} \% \text {-always similar to PLA) }\end{array}$ & [141] \\
\hline & $\begin{array}{l}\text { Solution mixing in chloroform, } \\
\text { drying and compression molding } \\
\left(180^{\circ} \mathrm{C}\right)\end{array}$ & $\begin{array}{l}\text { MWCNT, } \\
\text { MWCNT grafted with PLLA after reaction with } \\
\text { SOCl }_{2} \text { and ethylene glycol (MWCNT-g-PLLA) } \\
\text { Dimensions not given } \\
95 \% \text { purity }\end{array}$ & $\begin{array}{l}\text { MWCNT and } \\
\text { MWCNT-g-PLLA: } 0.1,0.2, \\
0.4,0.6,0.8,1.2\end{array}$ & $\begin{array}{l}\text { PLA/MWCNT: } \sigma=2 \times 10^{-13} \mathrm{~S} \mathrm{~m}^{-1} \\
(0.1-0.4 \mathrm{wt} \%), 3 \times 10^{-9} \mathrm{~S} \mathrm{~m}^{-1}(0.6 \mathrm{wt} \%) \text {, and } \\
2 \times 10^{-5} \mathrm{~S} \mathrm{~m}^{-1}(1.2 \mathrm{wt} \%) \\
\text { PLA/MWCNT-g-PLLA: } \sigma=2 \times 10^{-13} \mathrm{~S} \mathrm{~m}^{-1} \\
(0.1-0.4 \mathrm{wt} \%), 5 \times 10^{-13} \mathrm{~S} \mathrm{~m}^{-1}(0.6 \mathrm{wt} \%) \text {, and } \\
3 \times 10^{-8} \mathrm{~S} \mathrm{~m}^{-1}(1.2 \mathrm{wt} \%) \\
\text { Increases with filler amount }\end{array}$ & [142] \\
\hline & $\begin{array}{l}\text { Solution mixing in chloroform, } \\
\text { filtered, washed, dried under } \\
\text { vacuum, and compression } \\
\text { molded }\left(180^{\circ} \mathrm{C}, 500 \mathrm{psi}\right)\end{array}$ & $\begin{array}{l}\text { MWCNT, MWCNT-COOH (both as in [101]), } \\
\text { and MWCNT grafted with PLA chains of } \\
122-530 \mathrm{~g} \mathrm{~mol}^{-1} \text { by ring open polymerization } \\
\text { (MWCNT-g-PLA122-530). } \\
\mathrm{d}=10-15 \mathrm{~nm} \\
1=10-20 \mu \mathrm{m} \\
95 \% \text { purity }\end{array}$ & $\begin{array}{l}\text { MWCNT-COOH: } 1 \\
\text { MWCNT-g-PLA122-530: } 1\end{array}$ & $\begin{array}{l}\text { PLA/MWCNT-COOH: } \rho_{\square}=1 \times 10^{5} \Omega \mathrm{sq}^{-1} \\
\text { PLA/MWCNT-g-PLAA12-530: } \rho_{\square}=2 \times 10^{6} \text {, } \\
2 \times 10^{12} \text {, and } 1 \times 10^{12} \Omega / \mathrm{sq}(122,250, \\
\left.530 \mathrm{~g} \mathrm{~mol}^{-1}\right)\end{array}$ & [143] \\
\hline & $\begin{array}{l}\text { Sonication in THF, vacuum } \\
\text { drying, thermal compression }\end{array}$ & $\begin{array}{l}\text { MWCNT }(\mathrm{d}=8-15 \mathrm{~nm}, 1=50 \mu \mathrm{m}) \text { purified by } \\
\text { sonication with } \mathrm{H}_{2} \mathrm{SO}_{4} \text { and } \mathrm{HNO}_{3} \text { at } 50^{\circ} \mathrm{C} \text {, } \\
\text { filtration, and washing }\end{array}$ & $\begin{array}{l}\text { MWCNT } \\
\text { purified/non-purified: } 1 \\
3,5,7\end{array}$ & $\begin{array}{l}\text { PLA/MWCNT purified: } \sigma=4 \times 10^{-9}, \\
1 \times 10^{-9} \text {, and } 2 \times 10^{-6} \mathrm{~S} \mathrm{~m}^{-1}(1,5, \text { and } 7 \mathrm{wt} \%) \\
\text { PLA/MWCNT non-purified: } \sigma=7 \times 10^{-11}, \\
2 \times 10^{-8} \text {, and } 5 \times 10^{-8} \mathrm{~S} \mathrm{~m}^{-1}(1,5, \text { and } 7 \mathrm{wt} \%) \\
\text { Increases with filler amount }\end{array}$ & [88] \\
\hline & $\begin{array}{l}\text { Solution mixing in THF, vacuum } \\
\text { drying, thermal compression }\end{array}$ & $\begin{array}{l}\text { SWCNT }(\mathrm{d}<2 \mathrm{~nm}, 1=5-15 \mu \mathrm{m}, 95 \% \text { purity) } \\
\text { treated with } 3: 1 \mathrm{H}_{2} \mathrm{SO}_{4} / \mathrm{HNO}_{3}(\mathrm{~A}-\mathrm{SWCNT}) \text {, and } \\
\text { functionalized }(1: 2 \mathrm{v} / \mathrm{v}) \text { with 3-isocyanatoporpyl } \\
\text { triethoxysilane (IPTES) - A-SWCNT-Si }\end{array}$ & $\begin{array}{l}\text { SWCNT, A-SWCNT and } \\
\text { A-SWCNT-Si: } 0.1,0.3,0.5 \text {, } \\
1,3\end{array}$ & $\begin{array}{l}\text { PLA/SWCNT: } \sigma=2 \times 10^{-16}, 3 \times 10^{-9}, \text { and } \\
5 \times 10^{-8} \mathrm{~S} \mathrm{~m}^{-1}(0.3,1,3 \mathrm{wt} \%) \\
\mathrm{PLA} / \mathrm{A}-\mathrm{SWCNT}-\mathrm{Si}: \sigma=5 \times 10^{-15}, 5 \times 10^{-8}, \\
\text { and } 2 \times 10^{-6} \mathrm{~S} \mathrm{~m}^{-1}(0.3,1,3 \mathrm{wt} \%) \\
\text { Increases with filler amount }\end{array}$ & [144] \\
\hline
\end{tabular}


Table 2. Cont.

\begin{tabular}{|c|c|c|c|c|c|}
\hline Method & Procedure & CNT Characteristics & CNT Content (wt \%) & $\begin{array}{l}\text { Electrical Properties } \\
\sigma: \text { electrical conductivity } \\
\rho_{\square}: \text { sheet resistance } \\
\left(\mathrm{PLA} \sigma \approx 1 \times 10^{-16} \mathrm{~S} \mathrm{~m}^{-1},\right. \\
\left.\rho_{\square} \approx 5 \times 10^{12} \Omega \mathrm{sq}^{-1}\right)[106,122]\end{array}$ & References \\
\hline $\begin{array}{l}\text { Solution } \\
\text { mixing }\end{array}$ & $\begin{array}{l}\text { MWCNT-ox }\left(\mathrm{HCl}, 2 \mathrm{~h} \text { at } 25^{\circ} \mathrm{C}+\right. \\
\left.\mathrm{HNO}_{3}, 4 \mathrm{~h} \text { at } 110^{\circ} \mathrm{C}\right) \\
\text { Nanofibers (MWCNT-ox } \\
\text { sonicated in DMF } 2 \mathrm{~h}+\mathrm{SDS}, \\
\text { adding to PLA in dicloromethane, } \\
1 \mathrm{~h} \text { sonication before } \\
\text { electrospinning) }\end{array}$ & $\begin{array}{l}\text { MWCNT }(1=10-20 \mu \mathrm{m}, \mathrm{d}=10-20 \mathrm{~nm}) \\
\text { Nanofibers }(\text { PLA } \approx 400 \mathrm{~nm}, \text { PLA } / \text { MWCNT-ox } \\
\approx 250 \mathrm{~nm})\end{array}$ & $\begin{array}{l}\text { PLA/MWCNT-ox ( } 3 \mathrm{wt} \\
\% \text { ) random (R) and } \\
\text { aligned (A) nanofibers: } 1 \\
2,3,4,5 \mathrm{wt} \%\end{array}$ & $\begin{array}{l}\text { PLA/MWCNT-ox-R: } \rho_{\square}=1 \times 10^{4}, \\
5 \times 10^{2} \Omega \text { sq }^{-1}(3 \text { and } 5 \text { wt } \%) \\
\text { PLA/MWCNT-ox-A: } \rho_{\square}=5 \times 10^{3}, \\
1 \times 10^{2} \Omega \text { sq } \\
\text { Increases with both fillers amount }\end{array}$ & [183] \\
\hline \multirow{5}{*}{ Melt blending } & $\begin{array}{l}\text { Internal mixer }\left(180^{\circ} \mathrm{C}, 50 \mathrm{rpm},\right. \\
5 \text { min) with and without } \\
\text { transesterification with } \mathrm{Ti}(\mathrm{OBu})_{4} \\
\text { compression molding }\left(180^{\circ} \mathrm{C}\right)\end{array}$ & $\begin{array}{l}\text { MWCNT }(1=1-10 \mu \mathrm{m}) \text { functionalized with } \\
\mathrm{HNO}_{3}\left(120^{\circ} \mathrm{C}, 40 \mathrm{~min}\right)-\mathrm{MWCNT}-\mathrm{COOH} \text {, and } \\
\text { modified with DCC and stearyl alcohol } \\
\left.\text { (MWCNT-C } \mathrm{C}_{18} \mathrm{OH}\right)\end{array}$ & $\begin{array}{l}\text { PC: MWCNT/PLA } \\
\text { PC-18: } \\
\text { MWCNT-C18OH/PLA } \\
\text { PC-18T: } \\
\text { MWCNT-C18OH/PLA } \\
\text { transesterified } \\
0.5,1.5,3\end{array}$ & $\begin{array}{l}\text { PLA/PC: } \rho_{\square}=2 \times 10^{7}, 3 \times 10^{6} \text {, and } \\
3 \times 10^{5} \Omega \mathrm{sq}^{-1}(0.5,1.5,3 \mathrm{wt} \%) \\
\text { PLA/PC- } 18: \rho_{\square}=8 \times 10^{5}, 9 \times 10^{4} \text {, and } \\
1 \times 10^{-1} \Omega \mathrm{sq}^{-1}(0.5,1.5,3 \mathrm{wt} \%) \\
\text { PLA/PC- } 18 \mathrm{~T}: \rho_{\square}=5 \times 10^{12} \\
9 \times 10^{5} \text {, and } 9 \times 10^{-2} \Omega \mathrm{sq}^{-1}(0.5,1.5,3 \mathrm{wt} \%)\end{array}$ & [160] \\
\hline & $\begin{array}{l}\text { Twin-screw extruder }(180,215 \\
\text { and } 250^{\circ} \mathrm{C} ; 100,200 \text { and } 500 \mathrm{rpm} ; \\
5 \mathrm{~min}) \\
1 \mathrm{st} \text { - masterbatch production } \\
\text { 2nd-dilution of masterbatches } \\
\text { and composites production }\end{array}$ & $\begin{array}{l}\text { MWCNT } \\
\mathrm{d}=9.5 \mathrm{~nm} \\
1=1.5 \mu \mathrm{m} \\
90 \% \text { purity }\end{array}$ & MWCNT: $0.5,0.75,1,2$ & $\begin{array}{l}\sigma \text { is below } 2.5 \times 10^{-1} \mathrm{~S} \mathrm{~m}^{-1}(0.5-2 \mathrm{wt} \%) \\
\text { slightly decreasing with filler wt } \% \text { increase }\end{array}$ & [162] \\
\hline & $\begin{array}{l}\text { Twin-screw extrusion (150-190 } \\
\left.{ }^{\circ} \mathrm{C}, 100 \mathrm{rpm}\right) \text {, injection molding } \\
\left(160-190^{\circ} \mathrm{C}\right) \\
\text { High-crystalline PLA (HC-PLA) } \\
\text { and low-crystalline PLA } \\
\text { (LC-PLA) were tested }\end{array}$ & $\begin{array}{l}\text { MWCNT }(1=5-20 \mu \mathrm{m}, \mathrm{d}=40-60 \mathrm{~nm}) \\
\text { functionalized with maleic anhydride } \\
\text { (MWCNT-g-MA) at } 80^{\circ} \mathrm{C}, 4 \mathrm{~h} \text {, + benzoyl } \\
\text { peroxide }\end{array}$ & $\begin{array}{l}\text { LC-PLA/MWCNT, } \\
\text { HC-PLA/MWCNT and } \\
\text { MWCNT-g-MA: } 0.25,0.5 \\
0.75,1,2,4\end{array}$ & $\begin{array}{l}\text { LC-PLA/MWCNT: } \rho_{\square}=2 \times 10^{13}, 5 \times 10^{3} \text {, and } \\
5 \times 10^{2} \Omega \mathrm{sq}^{-1}(0.5,2,4 \mathrm{wt} \%) \\
\text { HC-PLA/MWCNT: } \rho_{\square}=1 \times 10^{14}, 9 \times 10^{10} \text {, and } \\
8 \times 10^{10} \Omega \mathrm{sq}^{-1}(0.5,2,4 \mathrm{wt} \%) \\
\text { LC-PLA/MWCNT-g-MA: } \rho_{\square}=3 \times 10^{2}, 2 \times 10^{2} \text {, } \\
\text { and } 7 \times 10^{1} \Omega \mathrm{sq}^{-1}(0.5,2,4 \mathrm{wt} \%)\end{array}$ & [163] \\
\hline & $\begin{array}{l}\text { Twin-screw extrusion }\left(180^{\circ} \mathrm{C},\right. \\
150 \mathrm{rpm}, 5 \mathrm{~min}) \text {, compression } \\
\text { molding at } 180^{\circ} \mathrm{C}\end{array}$ & $\begin{array}{l}\text { MWCNT } \\
\mathrm{d}=6-13 \mathrm{~nm}, 1=2.5-20 \mu \mathrm{m} \text {, specific surface area } \\
=220 \mathrm{~m}^{2} \mathrm{~g}^{-1} \\
\text { produced by CVD }\end{array}$ & MWCNT: $1.5,3,5$ & $\begin{array}{l}\sigma=1 \times 10^{-9}, 1 \times 10^{-2}, \text { and } 1 \mathrm{~S} \mathrm{~m}^{-1}(1.5,3 \\
5 \mathrm{wt} \%)\end{array}$ & [165] \\
\hline & $\begin{array}{l}\text { Twin-screw extruder }\left(180-220^{\circ} \mathrm{C},\right. \\
500 \mathrm{rpm}) \\
\text { Piston spinning }(20,50,100 \mathrm{~m} \\
\left.\min ^{-1}\right) \text { to produce micro-fibers } \\
\left(220^{\circ} \mathrm{C}, 3 \mathrm{~min}\right)\end{array}$ & $\begin{array}{l}\text { MWCNT } \\
\mathrm{d}=9.5 \mathrm{~nm} \\
1=1.5 \mu \mathrm{m} \\
90 \% \text { purity }\end{array}$ & MWCNT: 0.5, 1, 2, 3, 5 & $\begin{array}{l}\text { Extruded composites: } \sigma=4,14 \text {, and } 50 \mathrm{~S} \mathrm{~m}^{-1}(2, \\
3,5 \mathrm{wt} \%) \\
\text { Fibers }(3 \mathrm{wt} \%): \sigma=50,40 \text {, and } 1 \mathrm{~S} \mathrm{~m}^{-1} \\
\left.\text { (spinning speeds of } 20,50 \text {, and } 100 \mathrm{~m} \mathrm{~min}^{-1}\right)\end{array}$ & [184] \\
\hline
\end{tabular}


Table 2. Cont

\begin{tabular}{|c|c|c|c|c|c|}
\hline Method & Procedure & GBM Characteristics & GBM Content (wt \%) & $\begin{array}{l}\text { Electrical Properties } \\
\sigma: \text { electrical conductivity } \\
\rho_{\square}: \text { sheet resistance } \\
\left(\text { PLA } \sigma \approx 1 \times 10^{-16} \mathrm{~S} \mathrm{~m}^{-1},\right. \\
\left.\rho_{\square} \approx 5 \times 10^{12} \Omega \mathrm{sq}^{-1}\right)[106,122]\end{array}$ & References \\
\hline $\begin{array}{l}\text { Solution } \\
\text { mixing }\end{array}$ & $\begin{array}{l}\text { Sonication in DMF, coagulation } \\
\text { with methanol, drying, and } \\
\text { compression molding }\left(210^{\circ} \mathrm{C}\right)\end{array}$ & $\begin{array}{l}\text { GO: from graphite flakes (modified } \\
\text { Staudenmaier method) } \\
\text { rGO-p: GO + Polyvinylpyrrolidone (1:5), } \\
\text { sonication at } 60^{\circ} \mathrm{C} \\
\text { rGO-g: reduced by stirring with glucose in } \\
\text { ammonia solution at } 95^{\circ} \mathrm{C}, 60 \mathrm{~min} \\
\text { Dimension not given }\end{array}$ & $\begin{array}{l}\text { GO } \\
\text { rGO-p } \\
\text { rGO-g } \\
(0.5-2.5 \mathrm{vol} \%)\end{array}$ & $\begin{array}{l}\text { PLA/GO: } \sigma=\uparrow 6.5 \times 10^{-13} \mathrm{~S} \mathrm{~m}^{-1} \\
\text { PLA/rGO-p: } \sigma=\uparrow 4.7 \times 10^{-8} \mathrm{~S} \mathrm{~m}^{-1} \\
\text { PLA/rGO-g: } \sigma=2.2 \mathrm{~S} \mathrm{~m}^{-1} \\
\text { (for } 1.25 \text { vol \% for all) } \\
\text { Increases with filler amount }\end{array}$ & [155] \\
\hline Melt blending & $\begin{array}{l}\text { Twin-screw mixer }\left(175^{\circ} \mathrm{C},\right. \\
60 \mathrm{rpm}, 8 \mathrm{~min}) \text {, compression } \\
\text { molding at } 180{ }^{\circ} \mathrm{C}\end{array}$ & $\begin{array}{l}\text { GO prepared according to MHM and chemically } \\
\text { reduced to rGO. Thickness } 0.4-0.6 \mathrm{~nm} \text { and } \\
\text { lateral dimension } 0.1-0.5 \mathrm{~mm} \text {. }\end{array}$ & $\begin{array}{l}\text { rGO: } 0.02,0.04,0.06,0.2, \\
0.5,1,2\end{array}$ & $\begin{array}{l}\sigma=1 \times 10^{-13} \text { and } 1 \times 10^{-9} \mathrm{~S} \mathrm{~m}^{-1}(0.2 \text { and } \\
2 \mathrm{wt} \%) \\
\text { Increases with filler amount }\end{array}$ & [168] \\
\hline $\begin{array}{l}\text { In situ } \\
\text { polymerization }\end{array}$ & $\begin{array}{l}\text { Ring-opening melt } \\
\text { polymerization of lactide in } \\
\text { presence of } \operatorname{trGO}\end{array}$ & $\begin{array}{l}\text { GO prepared according to MHM and thermally } \\
\text { reduced to trGO } \\
\text { Dimensions not given }\end{array}$ & $\begin{array}{l}\text { TrGO: } 0.01,0.1,0.5,1,1.5 \\
2\end{array}$ & $\begin{array}{l}\sigma=5 \times 10^{-6} \text { and } 1.6 \times 10^{-2} \mathrm{~S} \mathrm{~m}^{-1 .}(1.5 \text { and } \\
2 \mathrm{wt} \%) \\
\text { Increases with filler amount }\end{array}$ & [176] \\
\hline
\end{tabular}


Considering $\rho_{\square}$, the best performance is obtained incorporating 3 wt $\%$ MWCNT- $\mathrm{C}_{18} \mathrm{OH}$ (MWCNT modified with DCC and stearyl alcohol) using and external mixer, followed by compression molding at $180^{\circ} \mathrm{C}$ during $5 \mathrm{~min}$, resulting in a $\rho_{\square}$ of $1 \times 10^{-1} \Omega \mathrm{sq}^{-1}$ [160]. This is the most effective modification performed, considering the sheet resistance values obtained with incorporation of the same amount of non-modified MWCNT, which was $3 \times 10^{5} \Omega \mathrm{sq}^{-1}$. For GBM, the higher $\sigma$ is $2.6 \times 10^{-4} \mathrm{~S} \mathrm{~m}^{-1}$, resultant from dispersion using an internal mixer at $180^{\circ} \mathrm{C}$, of $5 \mathrm{wt} \% \mathrm{PFG}$ (graphene nanoparticles functionalized with methylmethacrylate) [164]. For rGO, a non-functionalized GBM, the best conductivity value is obtained for $2 \mathrm{wt} \%$ incorporation in PLA using a twin-screw extruder and compression molding. The value obtained is of $1 \times 10^{-9} \mathrm{~S} \mathrm{~m}^{-1}$, being higher than for the other concentrations tested. It can be compared, for example, with a $\sigma$ of $1 \times 10^{-13} \mathrm{~S} \mathrm{~m}^{-1}$ for $0.2 \mathrm{wt} \%$ [168]. In most works evaluated, electrical properties improve with the increase of filler amount.

In situ polymerization is the least explored technique, despite interesting results being obtained by Yang et al. [176], which incorporate $0.01-2 \mathrm{wt} \%$ trGO (thermally reduced) in PLA by ring-opening melt polymerization of L-lactide in presence of the filler. As example, $\sigma$ obtained is $5 \times 10^{-6}$ and $1.6 \times 10^{-2} \mathrm{~S} \mathrm{~m}^{-1}$ for 1.5 and $2 \mathrm{wt} \%$, respectively.

An interesting study by Chiu et al. [88], shows that purification of MWCNT by sonication with strong acids improved fillers compatibility and dispersibility in PLA, resulting in better electrical conductivity. The values of $\sigma$ for incorporations of $7 \mathrm{wt} \%$ are $5 \times 10^{-8}$ and $2 \times 10^{-6} \mathrm{~S} \mathrm{~m}^{-1}$, respectively for non-purified and purified MWCNT. Purification introduced polar functional groups on the CNT surface, allowing better dispersion, which resulted in more deagglomerated particles that formed a wider conductive network on PLA matrix.

\subsection{Thermal Properties}

Several works studied thermal properties of PLA containing CBN. CNT incorporations range from 0.01 to $15 \mathrm{wt} \%$, while for GBM lower amounts are needed 0.01-2 wt \% (Table 3). However, for both CBN, slight or no changes are observed in the composites' thermal properties, especially when low fillers amounts are used [135,146,156,157,160-162,167]. The most frequently used techniques to evaluate thermal properties in polymer composites are thermogravimetric analysis (TGA), differential scanning calorimetry (DSC), and dynamic mechanical analysis (DMA). TGA allows determination of thermal degradation temperatures $\left(T_{d}\right)$ and DSC and DMA phase transition temperatures $\left(T_{g}\right.$-glass transition temperature, $T_{m}$-melting temperature, and $T_{c}$-cold crystallization temperature).

A positive deviation in $T_{d}$ is expected when there is good compatibility between CBN and the polymer matrix, combined with good dispersion of the fillers. This leads to restriction of PLA's chains motions, delaying thermal decomposition. Also, CBN can induce the formation of a crystallization region on their surfaces, which absorbs some heat as temperature of the composite increases. However, the incorporation of too high amounts of $\mathrm{CBN}$ can lead to the formation of agglomerates, which represent structural defects in the matrix, decreasing thermal stability [145]. Some works also attribute improvements in thermal stability to the barrier effect caused by the CBN, which creates a "tortuous path" delaying permeation of oxygen and the escape of volatile degradation products, and also to char formation $[146,150,167]$. Increases in $T_{g}$ are usually also associated with good interaction between CBN and polymer matrix, leading to constraint of PLA's molecular mobility by hydrogen bonding and electrostatic attraction $[139,140,146,150] . T_{m}$ increases are usually attributed to a nucleation effect caused by the CBN, which increases the degree of crystallinity $[146,150,176]$. For the same reason, $T_{\mathcal{C}}$ usually decreases with CBN incorporation $[141,146,153,162,170,176]$.

When using solution mixing, the highest variation in terms of $T_{g}$ is an increase of $10{ }^{\circ} \mathrm{C}$, obtained using $1 \mathrm{wt} \%$ MWCNT purified by treatment with strong acids. Comparing with non-purified filler at the same loading, the increase is $5{ }^{\circ} \mathrm{C}$ higher. This is explained by purified MWCNT having stronger interfacial interactions with PLA matrix, imposing increased restriction to the mobility of macromolecular chains, and therefore rising $T_{g}$. Also, $T_{d}$ (decomposition temperature) presents an increase of $10{ }^{\circ} \mathrm{C}$ for purified materials [88]. For $T_{m}$, the higher increase is of $16{ }^{\circ} \mathrm{C}$ for 0.3 and 
$1 \mathrm{wt} \%$ MWCNT-PCL (functionalized with poly(caprolactone)) incorporated in PLA aligned fibers by sonication in dichloromethane and electrospinning. Also, $T_{c}$ decreases more than $10^{\circ} \mathrm{C}$, due to MWCNT inducing heterogeneous crystallization [145]. However, the higher decrease in $T_{c}\left(<20^{\circ} \mathrm{C}\right)$, is obtained by Moon et al. [138], with the incorporation of 3-10 wt \% MWCNT, with a length of about $2000 \mu \mathrm{m}$. In literature, the degradation temperatures of the polymeric materials determined by TGA are presented in different terms. For example, as $T_{d i}$ (beginning of thermal degradation), $T_{d 5}$ (decomposition temperature for $5 \mathrm{wt} \%$ loss), and $T_{d 50}$ (decomposition temperature for $50 \%$ weight loss). For $T_{d i}$, the highest increase is of $20^{\circ} \mathrm{C}$, obtained incorporating $2.5 \mathrm{wt} \% \mathrm{MWCNT}-\mathrm{COOH}$ (carboxylated with strong acids) by sonication in PLA dispersed in dichloromethane and THF, followed by vacuum drying and compression molding [146]. Considering $T_{d 50}$, the best result is an increase of $1-3{ }^{\circ} \mathrm{C}$, in a work above described [145].

GBM incorporation also induces changes on thermal properties of PLA. For $T_{g}$, an increase of $7{ }^{\circ} \mathrm{C}$ was obtained sonicating $0.4 \mathrm{wt} \%$ GNP in PLA films prepared by solvent casting [135]. The highest increases in $T_{m}$ have been of $5{ }^{\circ} \mathrm{C}$, for samples obtained by compression molding of PLA with $0.5 \mathrm{wt} \%$ GO grafted with PLA, produced by vacuum drying a dispersion in chloroform [150]. Significant decrease in $T_{c}$, of $20{ }^{\circ} \mathrm{C}$, is observed for PLA with $2 \mathrm{wt} \% \mathrm{GO}$, obtained by solvent mixing [153]. Thermal stability of PLA has been shown to improve with addition of GBM. $2 \mathrm{wt} \%$ GONSs (graphene oxide nanosheets) increases $T_{d i}$ by $16^{\circ} \mathrm{C}$ in samples produced by solvent mixing [156]. Also, $T_{d 5}$ is increased by $11^{\circ} \mathrm{C}$ sonication of $0.2 \mathrm{wt} \%$ GNSs (graphene nanosheets) in PLA dispersed in DMF, dried under vacuum to produce composites [152]. Finally, $T_{d \max }$ ( $T$ of maximum degradation rate) increases $33^{\circ} \mathrm{C}$ for PLA filled with TRG, produced by solution mixing [154]. Chemical modifications of MWCNT are reported to increase thermal properties of the composites. For example, directly comparing with PLA/MWCNT(non-modified), the incorporation of $1 \mathrm{wt} \%$ MWCNT grafted with PLA in the same PLA matrix, results in increases of about $3{ }^{\circ} \mathrm{C}$ in $T_{g}$ and decreases of $9{ }^{\circ} \mathrm{C}$ in $T_{c}$ [141]. Treatment with strong acids followed by silanization of SWCNT [144], which are incorporated in PLA at loading ranging from 0.1 and $3 \mathrm{wt} \%$, results in increases of about $5{ }^{\circ} \mathrm{C}$ in $T_{g}$.

Concerning composites produced by melt-blending, the highest increases in $T_{g}$ are of $5-6{ }^{\circ} \mathrm{C}$, for PLA micro-fibers with $3 \mathrm{wt} \%$ MWCNT to PLA [184]. Also, $T_{c}$ is observed to decrease at most $12{ }^{\circ} \mathrm{C}$ with incorporation of 0.5 and $2 \mathrm{wt} \%$ MWCNT [170]. Chieng et al. [167], study on the thermal properties of PLA/PEG (9:1) blends with addition of 0.1-1 wt \% GNP, reveals no variations on $T_{g}, T_{m}$, and $T_{c}$. However, $T_{d i}, T_{\max }$, and $T_{50}$, increase by 56,53 , and $44{ }^{\circ} \mathrm{C}$, respectively, for $0.5 \mathrm{wt} \%$ loadings. In situ polymerization of L-lactide in presence of TRG in amounts from 0.01 to $2 \mathrm{wt} \%$ result in considerable increases on $T_{g}, T_{m}$, and $T_{d \max }$. For example, at $2 \mathrm{wt} \%$ loading, increases of 5, 14, and $18^{\circ} \mathrm{C}$ are obtained, respectively [176]. In a different work reporting in situ polymerization of L-lactide, covalent functionalization of GO with both 1,4-butanediol, and polyhedral silsesquioxane results in increases in $T_{g}\left(18,20^{\circ} \mathrm{C}\right), T_{c}\left(15,8{ }^{\circ} \mathrm{C}\right), T_{m}\left(7,5^{\circ} \mathrm{C}\right)$, and $T_{d 5}\left(23,11^{\circ} \mathrm{C}\right)$ comparing with PLA/GO composites at $1 \mathrm{wt} \%$ loadings [177]. 
Table 3. Thermal properties of PLA/CBN composites in comparison with non-modified PLA. Production methods and CBN characteristics.

\begin{tabular}{|c|c|c|c|c|c|}
\hline Method & Procedure & CNTs Characteristics & CNTs Content (wt \%) & Thermal Properties Relative to Neat Polymer & References \\
\hline \multirow{6}{*}{ Solution mixing } & $\begin{array}{l}\text { Sonication in chloroform, drying and } \\
\text { compression molding }\left(200^{\circ} \mathrm{C}, 150 \mathrm{Kgf} \mathrm{cm}^{-2} \text {, }\right. \\
15 \mathrm{~min})\end{array}$ & $\begin{array}{l}\text { MWCNT } \\
\text { Diameter }(\mathrm{d}) \text { not given } \\
\text { Length }(\mathrm{l}) \approx 2000 \mu \mathrm{m}\end{array}$ & MWCNT: $0.5,3,5,10$ & $\begin{array}{l}T_{g} \text { (glass transition) } \downarrow 1-4^{\circ} \mathrm{C}(3,5 \mathrm{wt} \%) \text { and }= \\
(10 \mathrm{wt} \%) \\
T_{c} \text { (crystallization) } \downarrow>20^{\circ} \mathrm{C}(3,5,10 \mathrm{wt} \%) \\
\mathrm{T}_{\mathrm{m}} \text { (melting) }=(3,5,10 \mathrm{wt} \%) \\
T_{d} \text { (degradation) } \uparrow 10-20^{\circ} \mathrm{C}(3,5,10 \mathrm{wt} \%)\end{array}$ & [138] \\
\hline & Sonication in chloroform, film casting & $\begin{array}{l}\text { Unzipped CNT (uCNT) } \\
\mathrm{d}=30 \mathrm{~nm} \\
1=10 \mu \mathrm{m} \\
95 \% \text { purity }\end{array}$ & uCNT: 1, 2, 3, 4, 5 & $\begin{array}{l}T_{g} \uparrow 7,8^{\circ} \mathrm{C}(3,5 \mathrm{wt} \%) \\
T_{m} \uparrow 5,3^{\circ} \mathrm{C}(3,5 \mathrm{wt} \%)\end{array}$ & [139] \\
\hline & $\begin{array}{l}\text { PLA was modified with benzoyl chloride } \\
\text { and pyridine (PLAm), then acid chloride } \\
\text { groups were added by reaction with thionyl } \\
\text { chloride and triethylamine, then fMWCNT } \\
\text { were added and the mixture centrifuged and } \\
\text { filtered to remove excess filler and salts. } \\
\text { Finally, sonication in chloroform and film } \\
\text { casting was performed }\end{array}$ & $\begin{array}{l}\text { MWCNT functionalized with } \mathrm{COOH} \text { using Fenton } \\
\text { reactant and then reacted with } \mathrm{SOCl}_{2} \text { and ethylene } \\
\text { glycol (fMWCNT). } \\
\mathrm{d}=9.5 \mathrm{~nm} \\
l=1.5 \mu \mathrm{m} \\
95 \% \text { purity }\end{array}$ & Not clear & $\begin{array}{l}T_{g}(\tan \delta) \uparrow 9{ }^{\circ} \mathrm{C} \\
T_{d i}\left(\text { beginning of thermal degradation) } \uparrow 80^{\circ} \mathrm{C}\right.\end{array}$ & [140] \\
\hline & $\begin{array}{l}\text { Sonication in chloroform, coagulation with } \\
\text { methanol, filtration, vacuum drying, and } \\
\text { compression molding }\left(180^{\circ} \mathrm{C}\right)\end{array}$ & $\begin{array}{l}\text { MWCNT (thermal CVD, } \mathrm{d}=10-15 \mathrm{~nm}, 1=10-20 \mu \mathrm{m}, \\
95 \% \text { purity) } \\
\mathrm{MWCNT} \text { carboxyl-functionalized (MWCNT-COOH) } \text { by } \mathrm{H}_{2} \mathrm{SO}_{4} 1: 3 \mathrm{HNO}_{3}, 3 \mathrm{~h}, 120^{\circ} \mathrm{C} \\
\mathrm{MWCNT} \text { grafted with PLA (MWCNT-g-PLA): } \text { MWCNT-COOH + L-lactide, } 12 \mathrm{~h}, 1500^{\circ} \mathrm{C},+ \text { tin(II) } \\
\text { chloride, } 20 \mathrm{~h}, 180^{\circ} \mathrm{C} \text {, under vacuum, filtration, } \\
\text { vacuum drying }\end{array}$ & $\begin{array}{l}\text { MWCNT: } 1 \\
\text { MWCNT-COOH: } 1 \\
\text { MWCNT-g-PLA: } 0.1 \\
0.2,0.5,1,5\end{array}$ & $\begin{array}{l}\text { No significant changes in } T_{m} \text { for all materials } \\
\text { PLA/MWCNT: } \\
T_{g} \uparrow 3, T_{c} \downarrow 3^{\circ} \mathrm{C}(1 \mathrm{wt} \%) \\
\text { PLA/MWCNT-COOH: } \\
T_{g} \uparrow 2, T_{c} \downarrow 3^{\circ} \mathrm{C}(1 \mathrm{wt} \%) \\
\text { PLA/MWCNT-g-PLA: } \\
T_{g} \uparrow 5-6 T_{c} \uparrow 1 \downarrow 2,6,12,19^{\circ} \mathrm{C}(0.1,0.2,0.5,1,5 \mathrm{wt} \%)\end{array}$ & [141] \\
\hline & $\begin{array}{l}\text { Sonication in dichloromethane, } \\
\text { electrospinning }\end{array}$ & $\begin{array}{l}\text { MWCNT ( } \mathrm{d}=8-15 \mathrm{~nm}, \mathrm{~L}-\text { not given, } 95 \% \text { purity) } \\
\text { were functionalized with -COOH by } \mathrm{H}_{2} \mathrm{SO}_{4} \text { and } \\
\mathrm{HNO}_{3}(3: 1) \text {. Then, MWCNT-NH } \\
\text { reacting MWCNT-COOH with } \\
\mathrm{N}, \mathrm{N}^{\prime} \text {-dicyclohexylcarbodiimide (DCC). MWCNT-PCL } \\
\text { were produced reacting } 1 \mathrm{~g} \text { MWCNT-NH } \\
\text { and } 20 \mathrm{~g} \text { DCC }\end{array}$ & $\begin{array}{l}\text { MWCNT-PCL }(0.3,0.5 \\
1,3) / \text { PLA aligned } \\
\text { composite fibers }\end{array}$ & $\begin{array}{l}T_{d 50}(50 \% \text { weight loss }) \uparrow 1-3^{\circ} \mathrm{C}(0.3,1 \mathrm{wt} \%) \\
T_{g}=(0.3,1 \mathrm{wt} \%) \\
T_{m} \uparrow 16^{\circ} \mathrm{C}(0.3,1 \mathrm{wt} \%) \\
T_{c} \downarrow 13{ }^{\circ} \mathrm{C} \text { and } 12{ }^{\circ} \mathrm{C}(0.3,1 \mathrm{wt} \%)\end{array}$ & [145] \\
\hline & $\begin{array}{l}\text { Sonication in THF, vacuum drying, thermal } \\
\text { compression }\end{array}$ & $\begin{array}{l}\text { MWCNT }(\mathrm{d}=8-15 \mathrm{~nm}, 1=50 \mu \mathrm{m}) \text { purified by } \\
\text { sonication with } \mathrm{H}_{2} \mathrm{SO}_{4} \text { and } \mathrm{HNO}_{3} \text { at } 50^{\circ} \mathrm{C} \text {, filtration, } \\
\text { and washing }\end{array}$ & $\begin{array}{l}\text { MWCNT } \\
\text { purified/non-purified: } \\
1,3,5,7\end{array}$ & $\begin{array}{l}\text { PLA/MWCNT non-purified: } T_{g} \uparrow 5-6{ }^{\circ} \mathrm{C} \\
(1,3,5,7 \mathrm{wt} \%) \\
\text { PLA/MWCNT purified: } T_{g} \uparrow 10,7,5,5^{\circ} \mathrm{C}(1,3,5, \\
7 \mathrm{wt} \%) \\
\text { PLA/MWCNT non-purified vs. purified: } T d \uparrow 10,11 \text {, } \\
7,8^{\circ} \mathrm{C}(1,3,5,7 \mathrm{wt} \%)\end{array}$ & {$[88]$} \\
\hline
\end{tabular}


Table 3. Cont

\begin{tabular}{|c|c|c|c|c|c|}
\hline Method & Procedure & CNTs Characteristics & CNTs Content (wt \%) & Thermal Properties Relative to Neat Polymer & References \\
\hline \multirow[t]{2}{*}{ Solution mixing } & $\begin{array}{l}\text { Solution mixing in THF, vacuum drying, } \\
\text { thermal compression }\end{array}$ & $\begin{array}{l}\mathrm{SWCNT}(\mathrm{d}<2 \mathrm{~nm}, \mathrm{l}=5-15 \mu \mathrm{m}, 95 \% \text { purity) treated } \\
\text { with 3:1 } \mathrm{H}_{2} \mathrm{SO}_{4} / \mathrm{HNO}_{3} \text { (A-SWCNT), and } \\
\text { functionalized ( }(1: 2 v / v) \text { with 3-isocyanatoporpyl } \\
\text { triethoxysilane (IPTES) - A-SWCNT-Si }\end{array}$ & $\begin{array}{l}\text { SWCNT, A-SWCNT, } \\
\text { and A-SWCNT-Si: } 0.1 \text {, } \\
0.3,0.5,1,3\end{array}$ & $\begin{array}{l}T_{d 5}(5 \mathrm{wt} \% \text { loss) } \downarrow \text { for PLA } / \text { SWCNT (poor } \\
\text { interfacial interaction), = for PLA/A-SWCNT, and } \\
\text { A-SWCNT-Si } \\
T_{g} \text { : (higher that pure PLA) PLA } / \text { SWCNT }< \\
\text { PLA/A-SWCNT }<\text { PLA /A-SWCNT-Si (considering } \\
\text { all loadings, increases are below } 5{ }^{\circ} \mathrm{C} \text { ) }\end{array}$ & [144] \\
\hline & $\begin{array}{l}\text { Sonication in dichloromethane and THF, } \\
\text { vacuum drying, and compression molding } \\
\left(190^{\circ} \mathrm{C}\right)\end{array}$ & $\begin{array}{l}\text { MWCNT }(\mathrm{d}=9-20 \mathrm{~nm}, 1=5 \mu \mathrm{m}) \text { functionalized with } \\
\text { 3:1 } \mathrm{H}_{2} \mathrm{SO}_{4} / \mathrm{HNO}_{3}(\mathrm{MWCNT}-\mathrm{COOH})\end{array}$ & $\begin{array}{l}\text { MWCNT-COOH: } 0.5 \\
1,2.5\end{array}$ & $\begin{array}{l}T_{d i} \uparrow 10-20^{\circ} \mathrm{C}(0.5-2.5 \mathrm{wt} \%) \\
T_{g} \uparrow 0,1,2^{\circ} \mathrm{C}(0.5,1,2.5 \mathrm{wt} \%) \\
\left.T_{c} \uparrow 1,2,4^{\circ} \mathrm{C} 0.5,1,2.5 \mathrm{wt} \%\right) \\
\left.T_{m} \uparrow 3,4,5^{\circ} \mathrm{C} 0.5,1,2.5 \mathrm{wt} \%\right) \\
\end{array}$ & [146] \\
\hline \multirow{5}{*}{ Melt blending } & $\begin{array}{l}\text { Internal mixer }\left(180^{\circ} \mathrm{C}, 50 \mathrm{rpm}, 5 \mathrm{~min}\right) \text { with } \\
\text { and without transesterification with } \\
\mathrm{Ti}(\mathrm{OBu})_{4} \text {, compression molding }\left(180^{\circ} \mathrm{C}\right)\end{array}$ & $\begin{array}{l}\text { MWCNT }(\mathrm{l}=1-10 \mu \mathrm{m}) \text { functionalized with } \mathrm{HNO}_{3} \\
\left(120^{\circ} \mathrm{C}, 40 \mathrm{~min}\right)-\mathrm{MWCNT}-\mathrm{COOH} \text { and modified } \\
\left.\text { with DCC and stearyl alcohol (MWCNT- } \mathrm{C}_{18} \mathrm{OH}\right)\end{array}$ & $\begin{array}{l}\text { PC: MWCNT/PLA } \\
\text { PC-18: } \\
\text { MWCNT-C18OH/PLA } \\
\text { PC-18T: } \\
\text { MWCNT-C18OH/PLA } \\
\text { transesterified } \\
0.5,1.5,3\end{array}$ & $\begin{array}{l}\text { PLA/PC, PLA/PC-18-No change in } T_{m} \\
\text { PLA/PC-18T-2 melting peaks, } 1 \text { bellow } T_{m} \text { for } \\
\text { pristine PLA (low } M_{w} \text { PLA from transesterification), } \\
\text { other at the same } T_{m}\end{array}$ & [160] \\
\hline & $\begin{array}{l}\text { Sonication in THF, vacuum drying + } \\
\text { Microextruder }\left(180^{\circ} \mathrm{C}, 50 \mathrm{rpm}, 5 \mathrm{~min}\right)\end{array}$ & $\begin{array}{l}\text { MWCNT }(\mathrm{d}=9.5 \mathrm{~nm}, 1=1.5 \mu \mathrm{m}) \text { produced by } \\
\text { catalytic carbon vapor deposition (CCVD) }\end{array}$ & MWCNT: $0.1,1$ & $T_{g} \uparrow 1^{\circ} \mathrm{C}(0.1,1 \mathrm{wt} \%)$ & [161] \\
\hline & $\begin{array}{l}\text { Twin-screw extruder }\left(180,215 \text { and } 250{ }^{\circ} \mathrm{C} ;\right. \\
100,200 \text { and } 500 \mathrm{rpm} ; 5 \mathrm{~min}) \\
1 \mathrm{st}-\text { masterbatch production } \\
\text { 2nd-dilution of masterbatches and } \\
\text { composites production }\end{array}$ & $\begin{array}{l}\text { MWCNT } \\
\mathrm{d}=9.5 \mathrm{~nm} \\
1=1.5 \mu \mathrm{m} \\
90 \% \text { purity }\end{array}$ & $\begin{array}{l}\text { MWCNT: } 0.5,0.75,1 \\
2,7.5,15\end{array}$ & Similar $T_{g}(7.5,15 \mathrm{wt} \%)$ & [162] \\
\hline & $\begin{array}{l}\text { Twin-screw extruder }\left(210^{\circ} \mathrm{C}, 400 \mathrm{rpm}\right) \text {, } \\
\text { compression molding }\left(210^{\circ} \mathrm{C}\right)\end{array}$ & $\begin{array}{l}\text { MWCNT } \\
\mathrm{d}=5-20 \mathrm{~nm} \\
\mathrm{l}=10 \mu \mathrm{m} \\
\text { Specific surface area }=100-700 \mathrm{~m}^{2} \mathrm{~g}^{-1} \\
\text { CCVD }\end{array}$ & MWCNT: 0.5, 1, 2, 3, 5 & $\begin{array}{l}T_{g} \downarrow 1,2^{\circ} \mathrm{C}(0.5,1-5 \mathrm{wt} \%) \\
T_{c} \downarrow 12,10,12,7,6^{\circ} \mathrm{C}(0.5,1,2,3,5 \mathrm{wt} \%) \\
T_{m} \downarrow 1,2^{\circ} \mathrm{C}(0.5-3,5 \mathrm{wt} \%)\end{array}$ & [170] \\
\hline & $\begin{array}{l}\text { Twin-screw extruder }\left(180-220^{\circ} \mathrm{C}, 500 \mathrm{rpm}\right) \\
\text { Piston spinning to produce micro-fibers } \\
\left(220^{\circ} \mathrm{C}, 3 \mathrm{~min}\right)\end{array}$ & $\begin{array}{l}\text { MWCNT } \\
d=9.5 \mathrm{~nm} \\
1=1.5 \mu \mathrm{m} \\
90 \% \text { purity }\end{array}$ & MWCNT: $0.5,1,2,3,5$ & $\begin{array}{l}T_{g}: \text { pellet }=(3 \mathrm{wt} \%) \\
\text { Fibers } \uparrow 5-6^{\circ} \mathrm{C}(3 \mathrm{wt} \%)\end{array}$ & [184] \\
\hline
\end{tabular}


Table 3. Cont.

\begin{tabular}{|c|c|c|c|c|c|}
\hline Method & Procedure & GBM Characteristics & GBM Content (wt \%) & Thermal Properties Relative to Neat Polymer & References \\
\hline \multirow{7}{*}{ Solution mixing } & $\begin{array}{l}\text { Sonication in chloroform, casting and doctor } \\
\text { blading } \\
\text { GO was pre-dispersed in acetone while GNP } \\
\text { was directly dispersed in chloroform }\end{array}$ & $\begin{array}{l}\text { GNP grade } \mathrm{M} \text { (commercial product) } \\
\mathrm{t}=6-8 \mathrm{~nm}, \mathrm{~d} \approx 5 \mu \mathrm{m} . \\
\mathrm{GO}(\mathrm{MHM}) \\
\mathrm{d} \approx 100 \mathrm{~nm}\end{array}$ & $\begin{array}{l}\text { GO and GNP: } 0.2,0.4, \\
0.6\end{array}$ & $\begin{array}{l}\text { PLA/GO: } T_{g} \uparrow 3,4,3^{\circ} \mathrm{C}(0.2,0.4,0.6 \mathrm{wt} \%) \\
\text { PLA/GNP: } T_{g} \uparrow 6,7,5^{\circ} \mathrm{C}(0.2,0.4,0.6 \mathrm{wt} \%) \\
\text { Similar } T_{m} \text { for both GO and GNP }\end{array}$ & [135] \\
\hline & $\begin{array}{l}\text { Sonication in chloroform, filtration, vacuum } \\
\text { drying, compression molding }\left(170^{\circ} \mathrm{C},\right. \\
10 \mathrm{~min})\end{array}$ & $\begin{array}{l}\mathrm{GO}(\text { from natural graphite, } \mathrm{MHM}+\text { lyophilization }) \mathrm{d} \\
\approx 300 \mathrm{~nm} \\
\mathrm{GO}-\mathrm{g} \text {-PLLA }\left(\mathrm{GO}+\mathrm{L} \text {-lactide }\left(\mathrm{Sn}(\mathrm{oct})_{2}\right) \text {, filtration, }\right. \\
\text { vacuum drying) }\end{array}$ & $\begin{array}{l}\text { GO and GO- } g \text {-PLLA: } \\
0.5\end{array}$ & $\begin{array}{l}\text { PLA/GO: } T_{g} \uparrow 6^{\circ} \mathrm{C} \\
T_{m} \uparrow 3^{\circ} \mathrm{C} \\
\text { PLA/GO-g-PLLA: } T_{g} \uparrow 6^{\circ} \mathrm{C} \\
T_{m} \uparrow 5^{\circ} \mathrm{C}\end{array}$ & [150] \\
\hline & $\begin{array}{l}\text { Stirring and sonication in DMF, coagulation } \\
\text { with methanol, filtration, and vacuum } \\
\text { drying }\end{array}$ & $\begin{array}{l}\text { GO (MHM) from expandable graphite, chemically } \\
\text { reduced with hydrazine, and lyophilized } \\
\text { (GNSs-solvent free graphene nanosheets) } \\
\mathrm{t}<1 \mathrm{~nm}, \mathrm{~d}<50 \mathrm{~nm}\end{array}$ & GNSs: 0.2 & $T_{d 5} \uparrow 11^{\circ} \mathrm{C}$ & [152] \\
\hline & $\begin{array}{l}\text { Sonication in DMF, film casting, vacuum } \\
\text { drying }\end{array}$ & $\begin{array}{l}\text { GO prepared according to Staudenmaier method } \\
\left(\mathrm{H}_{2} \mathrm{SO}_{4}+\mathrm{HNO}_{3}+\mathrm{KClO}_{3}\right) \\
\text { (dimensions not given) }\end{array}$ & GO: $0.5,1,2$ & $\begin{array}{l}(0.5,1,2 \mathrm{wt} \%) \\
T_{c} \downarrow 9,15,20^{\circ} \mathrm{C} \\
T_{g} \text { similar }\end{array}$ & [153] \\
\hline & $\begin{array}{l}\text { Sonication in DMF, coagulation with } \\
\text { methanol, drying, compression molding } \\
\left(185^{\circ} \mathrm{C}\right)\end{array}$ & $\begin{array}{l}\text { TRG (commercial product, } \mathrm{t}=\text { few layer, } \mathrm{d}=\text { hundreds } \\
\text { of nm) } \\
\text { TRG/PLA/Py-PLA: Py-PLA-OH (1-Pyrenemethanol } \\
\text { + L-lactide, Sn(oct) } 2)+ \text { TRG }(10: 1) \text { - sonication + } \\
\text { PLA—coagulation and drying }\end{array}$ & $\begin{array}{l}\text { TRG and } \\
\text { TRG/Py-PLA-OH: } \\
0.25,1\end{array}$ & $\begin{array}{l}\text { PLA/TRG: } \\
T_{d 5} \downarrow 32^{\circ} \mathrm{C} \\
T_{d \max } \text { (max. degradation) } \uparrow 33^{\circ} \mathrm{C} \\
\text { PLA/TRG/PLA/Py-PLA: } \\
T_{d 5} \downarrow 2{ }^{\circ} \mathrm{C} \\
T_{d \max } \uparrow 25^{\circ} \mathrm{C} \\
\text { (loadings not clear) }\end{array}$ & [154] \\
\hline & $\begin{array}{l}\text { Sonication in DMF, coagulation with water, } \\
\text { vacuum drying, compression molding } \\
\left(200^{\circ} \mathrm{C}, 3 \mathrm{~min}\right)\end{array}$ & $\begin{array}{l}\text { Graphene oxide nanosheets-GONSs (MHM) from } \\
\text { expandable graphite } \\
(\mathrm{t}=\text { few layer, } \mathrm{d}=5-20 \mu \mathrm{m})\end{array}$ & GONSs: $0.25,0.5,1,2$ & $\begin{array}{l}(0.25,0.5,1,2 \mathrm{wt} \%) \\
T_{m 1} \downarrow 1,4,0,1^{\circ} \mathrm{C} \\
T_{m 2} \downarrow 0,1,1,1^{\circ} \mathrm{C} \\
T_{c} \downarrow 3,6,2,4{ }^{\circ} \mathrm{C} \\
T_{d i} \uparrow 2,6,11,16^{\circ} \mathrm{C} \\
\end{array}$ & [156] \\
\hline & $\begin{array}{l}\text { Sonication in DMF, film casting, vacuum } \\
\text { drying }\end{array}$ & $\begin{array}{l}\text { GNS (commercial product) } \\
\mathrm{t}=5-25 \mathrm{~nm}, \mathrm{~d}=0.5-20 \mu \mathrm{m}, \text { specific surface area }= \\
50 \mathrm{~m}^{2} \mathrm{~g}^{-1}\end{array}$ & GNS: 1 & $\begin{array}{l}\text { Similar } T_{g} \text { and } T_{m 1} \text { and } 2 \\
T_{c} \uparrow 3^{\circ} \mathrm{C}\end{array}$ & [157] \\
\hline
\end{tabular}


Table 3. Cont

\begin{tabular}{|c|c|c|c|c|c|}
\hline Method & Procedure & GBM Characteristics & GBM Content (wt \%) & Thermal Properties Relative to Neat Polymer & References \\
\hline Melt blending & $\begin{array}{l}\text { Internal mixer }\left(160^{\circ} \mathrm{C}, 25 \mathrm{rpm}, 10 \mathrm{~min}\right) \text {, } \\
\text { compression molding }\left(160^{\circ} \mathrm{C}, 10 \mathrm{~min}\right) \\
\text { (Polymer was PLA/PEG 9:1 blend })\end{array}$ & $\begin{array}{l}\text { GNP grade M15 (commercial product) } \\
\mathrm{t}=6-8 \mathrm{~nm}, \mathrm{~d} \approx 15 \mu \mathrm{m}\end{array}$ & $\begin{array}{l}\text { GNP-M15: 0.1, 0.3, 0.5, } \\
0.7,1\end{array}$ & $\begin{array}{l}\text { (relative to pristine PLA/PEG blend) } \\
(0.1,0.3,0.5,1 \mathrm{wt} \%) \\
T_{g} \downarrow 0,0,1,1 \\
T_{m} \uparrow 2,4 \downarrow 1,1 \\
T_{c} \uparrow 1,2,2,1 \\
T_{d i}, T_{d \max }, T_{50} \uparrow 56,53,44^{\circ} \mathrm{C}(0.5 \mathrm{wt} \%) \\
\end{array}$ & [167] \\
\hline \multirow[b]{2}{*}{$\begin{array}{c}\text { In situ } \\
\text { polymerization }\end{array}$} & $\begin{array}{l}\text { Melt ring-opening polymerization of } \\
\text { L-lactide in presence of TRG }\left(\mathrm{Sn}(\mathrm{oct})_{2}\right. \\
\left.170{ }^{\circ} \mathrm{C}, 4 \mathrm{~h}\right) \text {, filtration, vacuum drying }\end{array}$ & $\begin{array}{l}\text { Natural graphite }(\mathrm{MHM}+\text { lyophilization })-G O \\
\mathrm{GO} \text { thermal reduction }\left(1000^{\circ} \mathrm{C}, 1 \mathrm{~min}\right) \text { to } \mathrm{TRG} \\
\mathrm{t}=\text { few layers }\end{array}$ & $\begin{array}{l}\text { TRG: } 0.01,0.1,0.5,1 \\
1.5,2\end{array}$ & $\begin{array}{l}(0.01,0.1,0.5,1,1.5,2 \mathrm{wt} \%) \\
T_{g}=\uparrow 9,6,6,7,8,5^{\circ} \mathrm{C} \\
T_{m}=\uparrow 11,12,13,14,14,14{ }^{\circ} \mathrm{C} \\
T_{d \max }=\uparrow 4,13,10,11,16,18^{\circ} \mathrm{C}\end{array}$ & [176] \\
\hline & $\begin{array}{l}\text { Sonication of L-lactide + filler in toluene, } \\
\text { addition of Tin(II)-2-ethylhexanoate under } \\
\mathrm{N}_{2} \text {, stirring at } 110^{\circ} \mathrm{C}, 3 \text { days }\end{array}$ & $\begin{array}{l}\text { Expanded graphite (MHM) to GOGO-functionalized: } \\
\text { GO + TDI +1,4-butanediol, } 80^{\circ} \mathrm{C}, 24 \mathrm{~h} \\
\text { GO-g-POSS: GO + POSS-polyhedral oligomeric } \\
\text { silsesquioxane + DMAP-4-(dimethylaminopyridine) } \\
+ \text { EDC- } N \text {-(3-dimethylamino- } \\
\text { propyl- } \mathrm{N}^{\prime} \text {-ethylcarbodiimide), } 2 \text { days, room } \\
\text { temperature, } \mathrm{N}_{2} \\
\text { (dimensions not given) }\end{array}$ & $\begin{array}{l}\text { GO-functionalized, } \\
\text { GO-g-POSS, } \\
\text { GO+POSS (physical } \\
\text { mixture): } 1\end{array}$ & $\begin{array}{l}\text { PLA/GO-functionalized: } \\
T_{d 5} \uparrow 8, T_{g} \downarrow 8, T_{c} \uparrow 14, T_{m} \downarrow 2{ }^{\circ} \mathrm{C} \\
\text { PLA/GO-g-POSS: } \\
T_{d 5} \uparrow 31, T_{g} \uparrow 10, T_{c} \uparrow 29, T_{m} \uparrow 5^{\circ} \mathrm{C} \\
\text { PLA/GO+POSS: } \\
T_{d 5} \uparrow 19, T_{g} \uparrow 12, T_{\mathcal{c}} \uparrow 22, T_{m} \uparrow 3{ }^{\circ} \mathrm{C}\end{array}$ & [177] \\
\hline
\end{tabular}




\subsection{Biological Properties}

Most nanomaterials may present toxicity at concentrations above a certain threshold when in isolated form, i.e., when not incorporated in a polymer matrix [40,186]. Biocompatibility of the composites must be tested when considering uses as biomaterials. Table 4 shows that PLA/CBN composites (films and nanofibers) do not tend to decrease in vitro metabolic activity of several cell types, or cause increases up to $40 \%$ until $72 \mathrm{~h}$ incubations. Also, the selection of production method used (melt blending or solvent mixing followed by casting, doctor blading, spin coating or electrospinning), does not seem to influence cell proliferation. For long term incubations, McCullen et al. [187] shows that scaffolds of PLA with $1 \mathrm{wt} \%$ MWNTs do not to influence metabolic activity of adipose-derived human mesenchymal stem cells (hMSCs) at 7 days. At 14 days, cells present increased metabolic activity and longitudinal alignment induced by the scaffolds. Sherrell et al. [188] reports PLGA (1:1) with a surface layer of graphene applied by CVD to increase PC-12 cells average length of neurites by 2.5 fold when electrical stimulated. Also, hemocompatibility improvements are reported with both incorporation of $0.4 \mathrm{wt} \%$ GNP by solvent mixing followed by doctor blading [149] and $4 \mathrm{wt} \%$ MWCNT by extrusion followed by injection molding [189] in PLA. In the last case, MWCNT alignment is associated with decreased platelet adhesion and activation. Thus, alignment seems to be generally benefit for biocompatibility. The bioeffectiveness of electrical stimulation together with nanofibers and its fillers alignment is confirmed by Shao et al. [183], which cultures osteoblasts at the surface of PLA/MWCNT-ox ( $3 \mathrm{wt} \%$ ) produced by solution mixing followed by electrospinning. They observe improvements in cell elongation $(190 \%)$ and metabolic activity $(20 \%)$ for random nanofibers $(\mathrm{d} \approx 250 \mathrm{~nm}$ ) under DC $100 \mu \mathrm{A}$, comparing to unstimulated controls. For aligned fibers the previous values increase by 90 and $40 \%$, respectively. The aspect ratio is higher for the latter, comparing with random stimulated fibers (Figure 5). Finally, An et al. [190] find that PLA composite films and nanofibers with $3 \mathrm{wt} \%$ PU and $5 \mathrm{wt} \%$ GO almost completely suppress Escherichia coli and Staphylococcus aureus growth after $24 \mathrm{~h}$, not affecting MC3T3-E1 cells metabolic activity. This effect is attributed to GO potentially inducing oxidative stress or physical disruption on bacteria.

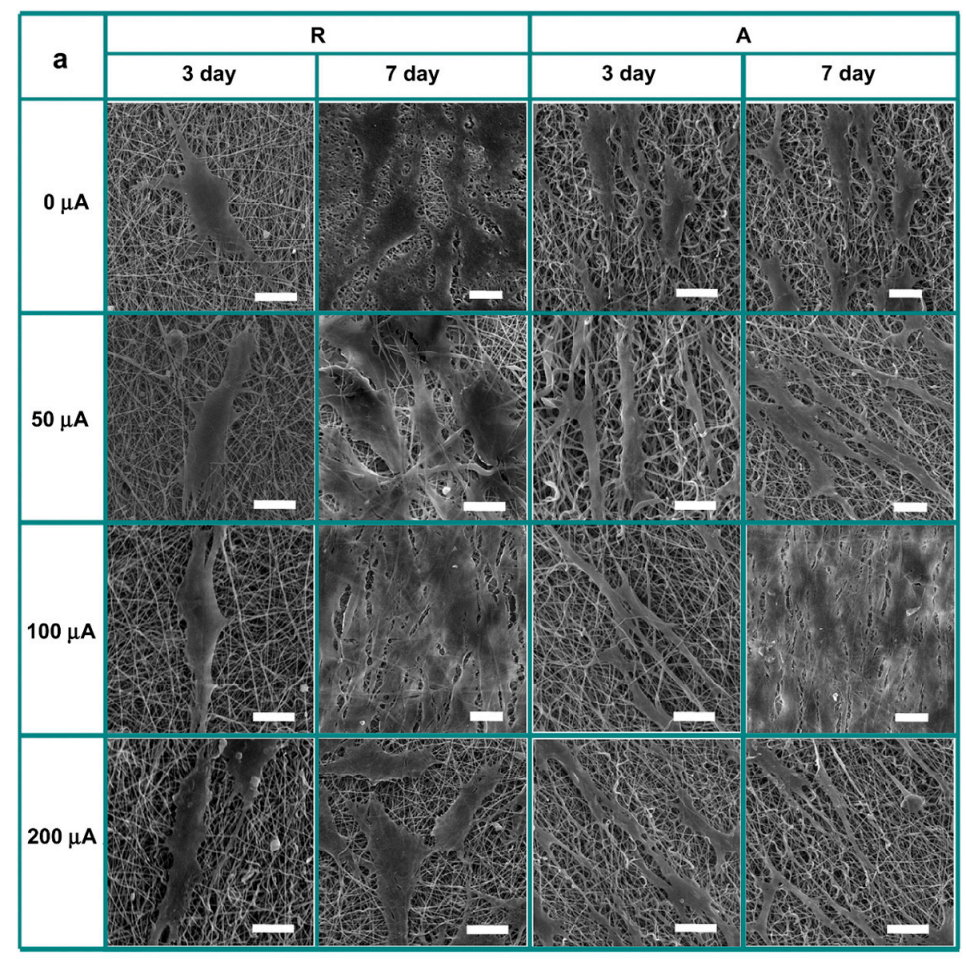

Figure 5. Cont. 

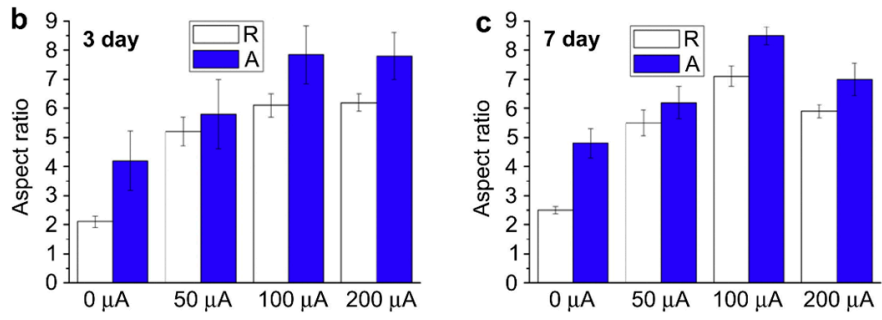

Figure 5. Scanning electron microscopy images of osteoblasts cultured on random (R) and aligned (A) nanofiber meshes of PLA/multi-walled carbon nanotubes (MWCNT)-ox $3 \mathrm{wt} \%$ produced by solution mixing followed by electrospinning, without or with electrical stimulation 0-200 $\mu \mathrm{A}$ (a); Osteoblast elongation is presented as the aspect ratio $(\mathbf{b}, \mathbf{c})$. Scale bars represent $30 \mu \mathrm{m}$ [191].

In an in vivo study, Kanczler et al. [192] observe that PLA-CB $0.1 \mathrm{wt} \%$ scaffolds seeded or not with fetal femur-derived cells, when implanted in a murine critical-size femur segmental defect model aid the regeneration of bone defect. Pinto et al. [193] report both PLA/GNP-M5 (2 wt \%) and CNT-COOH ( 0.3 and $0.7 \mathrm{wt} \%$ ) to be biocompatible, both in vitro and in vivo ( 2 weeks subcutaneous implantation in C57Bl/ 6 mice). Also, PLA/GNP-M5 and C $0.25 \mathrm{wt} \%$ composites have not release toxic products after 6 months degradation in phosphate-buffered saline at $37^{\circ} \mathrm{C}$ [180]. This is relevant considering that long-term biocompatibility must be assured for safe PLA/CBN composites implantation. 
Table 4. Biological properties of PLA/CBN composites in comparison with non-modified PLA. Production methods and CBN characteristics.

\begin{tabular}{|c|c|c|c|c|c|}
\hline Method & Procedure & CBN Characteristics & CBN Content (wt \%) & Biocompatibility Properties & References \\
\hline \multirow{9}{*}{$\begin{array}{c}\text { Solution } \\
\text { mixing }\end{array}$} & $\begin{array}{c}\text { GO-MHM } \\
\text { Nanofibers }(\mathrm{l}=11-14 \mu \mathrm{m}) \text { electrospinning }\end{array}$ & $\begin{array}{l}\mathrm{GO}(\text { thickness }(\mathrm{t})=1.5 \mathrm{~nm}, \\
\quad \text { length }(\mathrm{l}) \approx 1 \mu \mathrm{m})\end{array}$ & $\begin{array}{l}\text { PLGA }(1: 1) / G O 1 \text { and } \\
2 \mathrm{wt} \% \text { nanofibers }\end{array}$ & $\begin{array}{l}\text { Cell metabolic activity (MA): (PLGA }=100 \% \text {, } \\
\text { PLGA/GO } 1 \text { wt } \% \approx 102 \%, \text { PLGA } / \text { GO } 2 \text { wt } \% \approx \\
108 \%, 48 \mathrm{~h}) \\
(\text { PC } 12 \text { cells) }\end{array}$ & [191] \\
\hline & $\begin{array}{c}\text { GO-MHM } \\
\text { Films }(\mathrm{t} \approx 5 \mu \mathrm{m})-\text { spin coating }\end{array}$ & GO (not found) & PLGA (1:1)/GO films & $\begin{array}{l}\text { Cell MA: Small increase }(\approx 10 \%) \text { comparing to PLGA } \\
\text { for PLGA/GO } 2 \text { wt } \%(48 \text { h) (Hela cells) }\end{array}$ & {$[179]$} \\
\hline & $\begin{array}{l}\text { GO-MHM } \\
\text { Nanofibers (diameter }(\mathrm{d})=0.3-1.3 \mu \mathrm{m}) \\
\text { electrospinning }\end{array}$ & GO (few layer) & $\begin{array}{c}\mathrm{PLA} / \mathrm{HA}(10 \mathrm{wt} \%) / \mathrm{GO} \\
\text { nanofibers }\end{array}$ & $\begin{array}{c}\text { Cell MA: 1, } 2 \text { and } 5 \text { wt } \% \text { GO } \uparrow \text {, comparing to } \\
\text { PLA/HA }(24 \text { h) } \\
\text { Only nanofibers with } 5 \text { wt } \% \text { GO presented higher } \\
\text { MA than PLA/HA (48 h) } \\
\text { (MC3T3-E1 cells) }\end{array}$ & [185] \\
\hline & $\begin{array}{c}\text { GO-MHM } \\
\text { Films }(\mathrm{t}=25-65 \mu \mathrm{m}) \text { solvent mixing }+ \text { doctor } \\
\text { blading }\end{array}$ & $\mathrm{GO}(\mathrm{d} \approx 500 \mathrm{~nm})$ & $\begin{array}{l}\text { PLA/GO films } \\
\quad(0.4 \mathrm{wt} \%)\end{array}$ & \multirow{2}{*}{$\begin{array}{l}\text { Cell MA: No variations until } 48 \mathrm{~h} \text {, except for PLA/GO } \\
\text { after } 24 \mathrm{~h} \text { (more } 13 \% \text { than pristine PLA) (Mouse } \\
\text { embryo fibroblasts 3T3) } \\
\text { Hemocompatibility: Less human platelets activated in } \\
\text { PLA/GNP comparing with PLA in presence of } \\
\text { plasma proteins }\end{array}$} & \multirow[b]{2}{*}{ [149] } \\
\hline & $\begin{array}{c}\text { GNP-commercial product } \\
\text { Films }(\mathrm{t}=25-65 \mu \mathrm{m}) \text { solvent mixing }+ \text { doctor } \\
\text { blading }\end{array}$ & $\begin{array}{l}\text { GNP-M5 }(\mathrm{t} \approx 6-8 \mathrm{~nm} \\
1=5 \mu \mathrm{m})\end{array}$ & $\begin{array}{l}\text { PLA/GNP films } \\
\quad(0.4 \mathrm{wt} \%)\end{array}$ & & \\
\hline & $\begin{array}{c}\text { Graphene-CVD (chemical vapor } \\
\text { deposition) } \\
\text { Films }(\mathrm{t}=25-65 \mu \mathrm{m}) \text { solvent casting over } \\
\text { graphene }\end{array}$ & Graphene $(\mathrm{t}=2$ layers $)$ & $\begin{array}{l}\text { PLGA(1:1)/graphene } \\
\text { surface layer }\end{array}$ & $\begin{array}{l}\text { Cell MA: No significant changes until } 4 \text { days for } \\
\text { PC- } 12 \text { cells (rat adrenal gland pheochromocytoma) } \\
\text { Cell differentiation: with electrical stimulation the } \\
\text { average length of neurites increased 2.5-fold }\end{array}$ & [188] \\
\hline & $\begin{array}{c}\text { GO-MHM } \\
\text { Films (dimensions not found)—solvent } \\
\text { mixing }+ \text { solvent casting } \\
\text { Nanofibers }(\mathrm{d} \approx 1 \mu \mathrm{m}) \text { electrospinning }\end{array}$ & GO (not found) & $\begin{array}{l}\mathrm{PLA} / \mathrm{PU}(3 \mathrm{wt} \%) / \mathrm{GO} \\
\text { (5 wt \%) films and } \\
\text { nanofibers }\end{array}$ & $\begin{array}{c}\text { Cell proliferation: not decreased (MC3T3-E1 cells) } \\
\text { Antibacterial effect: E. coli and S. aureus growth } \\
100 \% \text { reduced at } 24 \mathrm{~h}\end{array}$ & [190] \\
\hline & $\begin{array}{c}\text { MWNTs }- \text { CVD } \\
\text { Scaffolds }(\mathrm{d}=0.7 \mu \mathrm{m}, \text { average porosity }= \\
87 \% \text {, void space }=89 \%) \text { - electrospinning }\end{array}$ & $\begin{array}{l}\text { MWNTs }(1=5-20 \mathrm{~mm} \\
\quad \mathrm{d}=5-15 \mathrm{~nm})\end{array}$ & $\begin{array}{l}\text { PLA/MWNTs }(1 \mathrm{wt} \%) \\
\text { scaffolds }\end{array}$ & $\begin{array}{l}\text { Cell MA: equal until day } 7 \text { and increased with } \\
\text { MWNTs at day } 14 \text { (hMSCs) } \\
\text { Cell morphology: MWNTs induced longitudinal } \\
\text { alignment on cells at day } 14\end{array}$ & {$[187,189]$} \\
\hline & $\begin{array}{c}\text { MWCNT-ox }\left(\mathrm{HCl}, 2 \mathrm{~h} \text { at } 25^{\circ} \mathrm{C}+\mathrm{HNO}_{3}, 4 \mathrm{~h}\right. \\
\left.\text { at } 110^{\circ} \mathrm{C}\right) \\
\text { Nanofibers (MWCNT-ox sonicated in DMF } \\
2 \mathrm{~h}+\text { SDS, adding to PLA in dicloromethane, } \\
\text { 1h sonication before electrospinning) } \\
\text { (PLA nanofibers, } \mathrm{d} \approx 400 \mathrm{~nm}, \\
\text { PLA/MWCNT-ox nanofibers, } \mathrm{d} \approx 250 \mathrm{~nm} \text { ) }\end{array}$ & $\begin{array}{l}\text { MWCNT }(1=10-20 \mu \mathrm{m} \\
\quad \mathrm{d}=10-20 \mathrm{~nm})\end{array}$ & $\begin{array}{l}\text { PLA/MWCNT-ox } \\
\text { (3 wt } \%) \text { random (R) and } \\
\text { aligned (A) nanofibers }\end{array}$ & $\begin{array}{c}\text { Cell MA: increased for osteoblasts at day } 3 \text { for } \\
\text { PLA/MWCNT-ox ( } 3 \mathrm{wt} \% \text { ) R-20\% and A- } 40 \% \text {, } \\
\text { under DC }=100 \mu \mathrm{A} \\
\text { Cell morphology: induced osteoblasts alignment at } \\
\text { day } 3 \text { for PLA/MWCNT-ox ( } 3 \mathrm{wt} \% \text { ) R- } \uparrow 190 \% \text { and } \\
\qquad \mathrm{A}-\uparrow 90 \% \text {, under DC }=100 \mu \mathrm{A}\end{array}$ & {$[183,187]$} \\
\hline
\end{tabular}


Table 4. Cont

\begin{tabular}{|c|c|c|c|c|c|}
\hline Method & Procedure & CBN Characteristics & CBN Content (wt \%) & Biocompatibility Properties & References \\
\hline \multirow{3}{*}{$\begin{array}{c}\text { Melt } \\
\text { blending }\end{array}$} & $\begin{array}{c}\text { MWNTs-CVD } \\
\text { Composites (dimensions not } \\
\text { found)—extrusion }+ \text { injection } \\
\text { moldingAligned composites-mechanical } \\
\text { stretching at } 90^{\circ} \mathrm{C}\end{array}$ & $\begin{array}{c}\text { MWNTs }(1=10-30 \mathrm{~mm} \\
\mathrm{d}=20-40 \mathrm{~nm})\end{array}$ & $\begin{array}{l}\text { PLA/MWNTs }(5,10, \\
15 \text { wt } \%) \text { composites }\end{array}$ & $\begin{array}{l}\text { Hemolysis: bellow standard permissible (5\%) in all } \\
\text { cases, decreases with MWNTs incorporation and } \\
\text { alignment } \\
\text { Kinetic clothing time: increases with MWNTs } \\
\text { incorporation and alignment (best was PLA/MWNTs } \\
5 \mathrm{wt} \% \text { which increased time by } 480 \%) \\
\text { Platelet adhesion and activation: decreases with } \\
\text { MWNTs incorporation and alignment }\end{array}$ & {$[183,189]$} \\
\hline & $\begin{array}{c}\text { GNP }(\text { commercial product }) \\
\text { Composites }(\mathrm{t} \approx 0.5 \mathrm{~mm}) \\
\text { Melt blending }+ \text { compression molding }\end{array}$ & $\begin{array}{l}\text { GNP-C }(\mathrm{t}=\text { up to } 2 \text { single } \\
\text { layers, } 1<2 \mu \mathrm{m}) \\
\text { GNP-M5 }(\mathrm{t} \approx 6-8 \mathrm{~nm} \\
1 \approx 5 \mu \mathrm{m})\end{array}$ & $\begin{array}{l}\text { PLA/GNP-C and M5 } \\
(0.25 \mathrm{wt} \%) \text { composites }\end{array}$ & $\begin{array}{l}\text { Comparing with PLA: similar cell adhesion and } \\
\text { growth at the surface } \\
\text { No release of toxic products after } 6 \text { months } \\
\text { degradation in phosphate-buffered saline at } 37^{\circ} \mathrm{C}\end{array}$ & [180] \\
\hline & $\begin{array}{c}\text { GNP (commercial product) } \\
\text { CNT-COOH-CVD, shortened, surface } \\
\text { oxidized } \\
\text { Composites }(\mathrm{t} \approx 0.5 \mathrm{~mm}) \\
\text { Melt blending }+ \text { compression molding }\end{array}$ & $\begin{array}{c}\text { GNP-M5 }(\mathrm{t} \approx 6-8 \mathrm{~nm} \\
1 \approx 5 \mu \mathrm{m}) \\
\text { CNT-COOH }(1<1 \mu \mathrm{m}, \mathrm{d} \\
=9.5 \mathrm{~nm},<8 \% \mathrm{COOH} \\
\text { content })\end{array}$ & $\begin{array}{l}\text { PLA/GNP-M5 }(2 \mathrm{wt} \%) \\
\text { PLA/CNT-COOH }(0.3 \\
\text { and } 0.7 \mathrm{wt} \%)\end{array}$ & $\begin{array}{l}\text { Biocompatible, both in vitro (human fibroblasts, } \\
\text { HFF-1) and in vivo } \\
\text { (2 weeks subcutaneous implantation in C57Bl/ } 6 \text { mice) }\end{array}$ & [193] \\
\hline $\begin{array}{c}\text { Laser } \\
\text { sintering }\end{array}$ & $\begin{array}{c}\text { CB (carbon black)—not found } \\
\text { Scaffolds (several shapes)—-surface selective } \\
\text { laser sintering }\end{array}$ & $\begin{array}{c}\text { (CB) Carbon black } \\
(\mathrm{d}=360 \mathrm{~nm}, \text { surface area } \\
\left.=100 \mathrm{~m}^{2} \mathrm{~g}^{-1}\right)\end{array}$ & $\begin{array}{l}\text { SSLS-PLA/CB } 0.1 \mathrm{wt} \% \\
\text { scaffolds }\end{array}$ & $\begin{array}{l}\text { SSLS-PLA/CB 0.1 wt \% scaffolds seeded or not with } \\
\text { fetal femur-derived cells aided regeneration of murine } \\
\text { bone defect }\end{array}$ & [192] \\
\hline
\end{tabular}




\section{Conclusions}

Both CNT and GBM nanofillers are effective at improving PLA thermo-mechanical and electrical properties. However, lower amounts of GBM (0.1-1 wt \%) are usually needed when comparing with CNT (0.25-5 wt \%). Melt-blending is less reported than solution mixing for production of PLA/CBN composites, maybe because it implies use of specialized equipment. Moreover, results show that melt blending suffers from some drawbacks, since viscous shear is less effective than solvent sonication for promoting exfoliation/deagglomeration of CBN. In situ polymerization is the least reported technique, with further research being needed to demonstrate its advantages over the previous production methods.

Surface modifications of CBN can be used to improve compatibility with a polymer matrix. Functionalization with carboxyls is the most common and effective procedure to improve CNT dispersibility and compatibility with PLA. Some authors refer that purification with strong acids introduces polar groups in the carbon surface, which results in positive interaction with PLA. Besides straightforward chemical oxidation of $\mathrm{CBN}$, other chemical modifications which lead to better performance after incorporation in PLA, comparing with non-modified CBN, include reaction with isocyanates, polyols, or silanes, and grafting with polymers (ethylene glycol, poly(caprolactone), poly(methyl methacrylate), poly(vinyl pyrrolidone), and PLA).

When comparing reduced and oxidized forms of GBM as PLA fillers, like rGO and GO, only in the case of increasing electrical conductivity the reduced forms show clearly better performance.

Based on the available data, no relation can be determined between CBN morphological properties (size, length, and diameter) and the composites performances.

The alignment of PLA/CNT fibers, has been shown to improve electrical conductivity. Electrical properties also improve with the increase of the amount of CBN incorporated.

Concerning biological properties, the composite production process does not influence cell metabolic activity, which does not decrease comparing to non-filled PLA. Furthermore, increases up to $40 \%$ in cell viability can be induced by GBM incorporation. Improvements in hemocompatibility are achieved with incorporation of both CNT and GBM. Also, both fiber/filler alignment and electrical stimulation, improve cell metabolic activity and elongation. Short term in vivo studies reveal PLA/CBN composites to be biocompatible, and no release of toxic degradation products is found up to 6 months in vitro degradation of PLA/GBM composites. Incorporation of GO has lead to suppression of Escherichia coli and Staphylococcus aureus growth, without compromising the composite biocompatibility. However, there is still no information on antimicrobial activity of these composites on other types of microorganisms or with other types of GBM. Also, long-term in vivo biocompatibility of PLA/CBN composites needs to be assured prior to their clinical use.

Some other relevant topics for future research include obtaining a better understanding of how the fillers physico-chemical properties, and their alignment inside the polymer matrix, affect the composites properties. In situ polymerization of PLA in presence of CBN is a not well developed topic, being worthwhile of further exploration due to the potential for optimization of the degree of interaction and dispersion of CBN in the polymer matrix. Mechanical milling is an increasingly interesting technique for mixing filler nanoparticles with a polymer matrix, but has not yet been reported for producing PLA/CBN composites. This is expected to change in the near future. Finally, emerging technologies, like 3D printing, will surely contribute to the conception of materials appropriate for the broad potential applications of PLA/CBN composites.

Acknowledgments: This work was financially supported by: Project POCI-01-0145-FEDER-006939 (Laboratory for Process Engineering, Environment, Biotechnology and Energy-LEPABE), Project POCI-01-0145-FEDER-007274 (Institute for Research and Innovation in Health Sciences), and Project PTDC/CTM-BIO/4033/2014 (NewCat), funded by FEDER funds through COMPETE2020-Programa Operacional Competitividade e Internacionalização (POCI) - and by national funds through FCT_Fundação para a Ciência e a Tecnologia; PhD grant SFRH/BD/ 86974/2012, funded by European Social Fund and Portuguese Ministry of Education and Science (MEC) through Programa Operacional Capital Humano (POCH). 
Author Contributions: Artur M. Pinto and Carolina Gonçalves have compiled the literature and written the text. Inês C. Gonçalves and Fernão D. Magalhães have revised the text and made suggestions concerning its structure and contents.

Conflicts of Interest: The authors declare no conflict of interest.

\section{References}

1. Oksman, K.; Skrifvars, M.; Selin, J.F. Natural fibers as reinforcement in polylactic acid (PLA) composites. Compos. Sci. Technol. 2003, 63, 1317-1324. [CrossRef]

2. Vaia, R.A.; Wagner, H.D. Framework for nanocomposites. Mater. Today 2004, 7, 32-37. [CrossRef]

3. Lasprilla, A.J.R.; Martinez, G.A.R.; Lunelli, B.H.; Jardini, A.L.; Maciel, R. Poly-lactic acid synthesis for application in biomedical devices-A review. Biotechnol. Adv. 2012, 30, 321-328. [CrossRef] [PubMed]

4. Vieira, A.C.; Vieira, J.C.; Ferra, J.M.; Magalhaes, F.D.; Guedes, R.M.; Marques, A.T. Mechanical study of PLA-PCL fibers during in vitro degradation. J. Mech. Behav. Biomed. 2011, 4, 451-460. [CrossRef] [PubMed]

5. Chang, J.H.; An, Y.U.; Sur, G.S. Poly(lactic acid) nanocomposites with various organoclays. I. Thermomechanical properties, morphology, and gas permeability. J. Polym. Sci. 2003, 41, 94-103. [CrossRef]

6. Mittal, V. Polymer layered silicate nanocomposites: A review. Materials 2009, 2, 992-1057. [CrossRef]

7. Raquez, J.M.; Habibi, Y.; Murariu, M.; Dubois, P. Polylactide (PLA)-based nanocomposites. Prog. Polym. Sci. 2013, 38, 1504-1542. [CrossRef]

8. Bafekrpour, E.; Salehi, M.; Sonbolestan, E.; Fox, B. Effects of micro-structural parameters on mechanical properties of carbon nanotube polymer nanocomposites. Sci. Iran. 2014, 21, 403-413.

9. Coleman, J.N.; Khan, U.; Gun'ko, Y.K. Mechanical reinforcement of polymers using carbon nanotubes. Adv. Mater. 2006, 18, 689-706. [CrossRef]

10. Fiedler, B.; Gojny, F.H.; Wichmann, M.H.G.; Nolte, M.C.M.; Schulte, K. Fundamental aspects of nano-reinforced composites. Compos. Sci. Technol. 2006, 66, 3115-3125. [CrossRef]

11. Pinto, A.M.; Goncalves, I.C.; Magalhaes, F.D. Graphene-based materials biocompatibility: A review. Colloid Surf. B 2013, 111, 188-202. [CrossRef] [PubMed]

12. Tjong, S.C. Structural and mechanical properties of polymer nanocomposites. Mater. Sci. Eng. R 2006, 53, 73-197. [CrossRef]

13. Xie, X.L.; Mai, Y.W.; Zhou, X.P. Dispersion and alignment of carbon nanotubes in polymer matrix: A review. Mater. Sci. Eng. R 2005, 49, 89-112. [CrossRef]

14. Pinto, A.M.; Martins, J.; Moreira, J.A.; Mendes, A.M.; Magalhaes, F.D. Dispersion of graphene nanoplatelets in poly(vinyl acetate) latex and effect on adhesive bond strength. Polym. Int. 2013, 62, 928-935. [CrossRef]

15. Pinto, A.M.; Goncalves, C.; Sousa, D.M.; Ferreira, A.R.; Moreira, J.A.; Goncalves, I.C.; Magalhaes, F.D. Smaller particle size and higher oxidation improves biocompatibility of graphene-based materials. Carbon 2016, 99, 318-329. [CrossRef]

16. Pinto, A.M.; Moreira, J.A.; Magalhaes, F.D.; Goncalves, I.C. Polymer surface adsorption as a strategy to improve the biocompatibility of graphene nanoplatelets. Colloid Surf. B 2016, 146, 818-824. [CrossRef] [PubMed]

17. Ge, C.C.; Li, Y.; Yin, J.J.; Liu, Y.; Wang, L.M.; Zhao, Y.L.; Chen, C.Y. The contributions of metal impurities and tube structure to the toxicity of carbon nanotube materials. NPG Asia Mater. 2012, 4, e32. [CrossRef]

18. Liu, X.; Guo, L.; Morris, D.; Kane, A.B.; Hurt, R.H. Targeted removal of bioavailable metal as a detoxification strategy for carbon nanotubes. Carbon 2008, 46, 489-500. [CrossRef] [PubMed]

19. Liu, Y.; Zhao, Y.L.; Sun, B.Y.; Chen, C.Y. Understanding the toxicity of carbon nanotubes. Acc. Chem. Res. 2013, 46, 702-713. [CrossRef] [PubMed]

20. Tejral, G.; Panyala, N.R.; Havel, J. Carbon nanotubes: Toxicological impact on human health and environment. J. Appl. Biomed. 2009, 7, 1-13.

21. Das, T.K.; Prusty, S. Graphene-based polymer composites and their applications. Polym. Plast. Technol. 2013, 52, 319-331. [CrossRef]

22. Huang, X.; Qi, X.Y.; Boey, F.; Zhang, H. Graphene-based composites. Chem. Soc. Rev. 2012, 41, $666-686$. [CrossRef] [PubMed]

23. Kotov, N.A. Materials science: Carbon sheet solutions. Nature 2006, 442, 254-255. [CrossRef] [PubMed] 
24. Kuilla, T.; Bhadra, S.; Yao, D.H.; Kim, N.H.; Bose, S.; Lee, J.H. Recent advances in graphene based polymer composites. Prog. Polym. Sci. 2010, 35, 1350-1375. [CrossRef]

25. Gupta, B.; Revagade, N.; Hilborn, J. Poly(lactic acid) fiber: An overview. Prog. Polym. Sci. 2007, 32, 455-482. [CrossRef]

26. Hu, Y.Z.; Daoud, W.A.; Cheuk, K.K.L.; Lin, C.S.K. Newly developed techniques on polycondensation, ring-opening polymerization and polymer modification: Focus on poly(lactic acid). Materials 2016, 9, 133. [CrossRef]

27. Lim, L.T.; Auras, R.; Rubino, M. Processing technologies for poly(lactic acid). Prog. Polym. Sci. 2008, 33, 820-852. [CrossRef]

28. Nampoothiri, K.M.; Nair, N.R.; John, R.P. An overview of the recent developments in polylactide (PLA) research. Bioresour. Technol. 2010, 101, 8493-8501. [CrossRef] [PubMed]

29. Rasal, R.M.; Janorkar, A.V.; Hirt, D.E. Poly(lactic acid) modifications. Prog. Polym. Sci. 2010, 35, 338-356. [CrossRef]

30. Pang, X.A.; Zhuang, X.L.; Tang, Z.H.; Chen, X.S. Polylactic acid (PLA): Research, development and industrialization. Biotechnol. J. 2010, 5, 1125-1136. [CrossRef] [PubMed]

31. Allen, M.J.; Tung, V.C.; Kaner, R.B. Honeycomb carbon: A review of graphene. Chem. Rev. 2010, 110, $132-145$. [CrossRef] [PubMed]

32. Balandin, A.A. Thermal properties of graphene and nanostructured carbon materials. Nat. Mater. 2011, 10, 569-581. [CrossRef] [PubMed]

33. Baughman, R.H.; Zakhidov, A.A.; de Heer, W.A. Carbon nanotubes-The route toward applications. Science 2002, 297, 787-792. [CrossRef] [PubMed]

34. Dreyer, D.R.; Park, S.; Bielawski, C.W.; Ruoff, R.S. The chemistry of graphene oxide. Chem. Soc. Rev. 2010, 39, 228-240. [CrossRef] [PubMed]

35. Lin, X.H.; Gai, J.G. Synthesis and applications of large-area single-layer graphene. RSC Adv. 2016, 6, 17818-17844. [CrossRef]

36. Nguyen, V.H. Recent advances in experimental basic research on graphene and graphene-based nanostructures. Adv. Nat. Sci. Nanosci. 2016, 7. [CrossRef]

37. Novoselov, K.S.; Fal'ko, V.I.; Colombo, L.; Gellert, P.R.; Schwab, M.G.; Kim, K. A roadmap for graphene. Nature 2012, 490, 192-200. [CrossRef] [PubMed]

38. Park, S.; Ruoff, R.S. Chemical methods for the production of graphenes. Nat. Nanotechnol. 2009, 4, $217-224$. [CrossRef] [PubMed]

39. Rao, C.N.R.; Sood, A.K.; Subrahmanyam, K.S.; Govindaraj, A. Graphene: The new two-dimensional nanomaterial. Angew. Chem. Int. Ed. 2009, 48, 7752-7777. [CrossRef] [PubMed]

40. Singh, V.; Joung, D.; Zhai, L.; Das, S.; Khondaker, S.I.; Seal, S. Graphene based materials: Past, present and future. Prog. Mater. Sci. 2011, 56, 1178-1271. [CrossRef]

41. Tasis, D.; Tagmatarchis, N.; Bianco, A.; Prato, M. Chemistry of carbon nanotubes. Chem. Rev. 2006, 106, 1105-1136. [CrossRef] [PubMed]

42. Thostenson, E.T.; Ren, Z.F.; Chou, T.W. Advances in the science and technology of carbon nanotubes and their composites: A review. Compos. Sci. Technol. 2001, 61, 1899-1912. [CrossRef]

43. Yang, F.; Wang, X.; Li, M.H.; Liu, X.Y.; Zhao, X.L.; Zhang, D.Q.; Zhang, Y.; Yang, J.; Li, Y. Templated synthesis of single-walled carbon nanotubes with specific structure. Acc. Chem. Res. 2016, 49, 606-615. [CrossRef] [PubMed]

44. Yeung, C.S.; Tian, W.Q.; Liu, L.V.; Wang, Y.A. Chemistry of single-walled carbon nanotubes. J. Comput. Theor. Nanosci. 2009, 6, 1213-1235. [CrossRef]

45. Zhang, F.; Hou, P.X.; Liu, C.; Cheng, H.M. Epitaxial growth of single-wall carbon nanotubes. Carbon 2016, 102, 181-197. [CrossRef]

46. Zhu, Y.W.; Murali, S.; Cai, W.W.; Li, X.S.; Suk, J.W.; Potts, J.R.; Ruoff, R.S. Graphene and graphene oxide: Synthesis, properties, and applications. Adv. Mater. 2010, 22, 3906-3924. [CrossRef] [PubMed]

47. Datta, R.; Henry, M. Lactic acid: Recent advances in products, processes and technologies—A review. J. Chem. Technol. Biotechnol. 2006, 81, 1119-1129. [CrossRef]

48. Jamshidian, M.; Tehrany, E.A.; Imran, M.; Jacquot, M.; Desobry, S. Poly-lactic acid: Production, applications, nanocomposites, and release studies. Compr. Rev. Food Sci. Food Saf. 2010, 9, 552-571. [CrossRef]

49. Garlotta, D. A literature review of poly(lactic acid). J. Polym. Environ. 2001, 9, 63-84. [CrossRef] 
50. Auras, R.; Lim, L.-T.; Selke, S.E.M.; Tsuji, H. Poly (lactic) acid: Synthesis, Structures, Properties, Processing, and Applications; John Wiley and Sons: San Francisco, CA, USA, 2010.

51. Drumright, R.E.; Gruber, P.R.; Henton, D.E. Polylactic acid technology. Adv. Mater. 2000, 12, $1841-1846$. [CrossRef]

52. Shogren, R.L.; Doane, W.M.; Garlotta, D.; Lawton, J.W.; Willett, J.L. Biodegradation of starch/polylactic acid/poly(hydroxyester-ether) composite bars in soil. Polym. Degrad. Stab. 2003, 79, 405-411. [CrossRef]

53. Zeng, J.B.; Li, K.A.; Du, A.K. Compatibilization strategies in poly(lactic acid)-based blends. RSC Adv. 2015, 5, 32546-32565. [CrossRef]

54. Semba, T.; Kitagawa, K.; Ishiaku, U.S.; Hamada, H. The effect of crosslinking on the mechanical properties of polylactic acid/polycaprolactone blends. J. Appl. Polym. Sci. 2006, 101, 1816-1825. [CrossRef]

55. Wang, H.; Sun, X.Z.; Seib, P. Mechanical properties of poly(lactic acid) and wheat starch blends with methylenediphenyl diisocyanate. J. Appl. Polym. Sci. 2002, 84, 1257-1262. [CrossRef]

56. Balakrishnan, H.; Hassan, A.; Wahit, M.U.; Yussuf, A.A.; Razak, S.B.A. Novel toughened polylactic acid nanocomposite: Mechanical, thermal and morphological properties. Mater. Des. 2010, 31, 3289-3298. [CrossRef]

57. Broz, M.E.; VanderHart, D.L.; Washburn, N.R. Structure and mechanical properties of poly(D,L-lactic acid)/poly(epsilon-caprolactone) blends. Biomaterials 2003, 24, 4181-4190. [CrossRef]

58. Abdelwahab, M.A.; Flynn, A.; Chiou, B.S.; Imam, S.; Orts, W.; Chiellini, E. Thermal, mechanical and morphological characterization of plasticized pla-phb blends. Polym. Degrad. Stab. 2012, 97, 1822-1828. [CrossRef]

59. Yew, G.H.; Yusof, A.M.M.; Ishak, Z.A.M.; Ishiaku, U.S. Water absorption and enzymatic degradation of poly(lactic acid)/rice starch composites. Polym. Degrad. Stab. 2005, 90, 488-500. [CrossRef]

60. Pellis, A.; Acero, E.H.; Ferrario, V.; Ribitsch, D.; Guebitz, G.M.; Gardossi, L. The closure of the cycle: Enzymatic synthesis and functionalization of bio-based polyesters. Trends Biotechnol. 2016, 34, 316-328. [CrossRef] [PubMed]

61. Gumel, A.M.; Annuar, M.S.M.; Heidelberg, T. Current application of controlled degradation processes in polymer modification and functionalization. J. Appl. Polym. Sci. 2013, 129, 3079-3088. [CrossRef]

62. Hoveizi, E.; Nabiuni, M.; Parivar, K.; Rajabi-Zeleti, S.; Tavakol, S. Functionalisation and surface modification of electrospun polylactic acid scaffold for tissue engineering. Cell Biol. Int. 2014, 38, 41-49. [CrossRef] [PubMed]

63. Kucharczyk, P.; Poljansek, I.; Sedlarik, V.; Kasparkova, V.; Salakova, A.; Drbohlav, J.; Cvelbar, U.; Saha, P. Functionalization of polylactic acid through direct melt polycondensation in the presence of tricarboxylic acid. J. Appl. Polym. Sci. 2011, 122, 1275-1285. [CrossRef]

64. Yuan, X.B.; Kang, C.S.; Zhao, Y.H.; Gu, M.Q.; Pu, P.Y.; Tian, N.J.; Sheng, J. Surface multi-functionalization of poly(lactic acid) nanoparticles and c6 glioma cell targeting in vivo. Chin. J. Polym. Sci. 2009, 27, 231-239. [CrossRef]

65. Iwatake, A.; Nogi, M.; Yano, H. Cellulose nanofiber-reinforced polylactic acid. Compos. Sci. Technol. 2008, 68, 2103-2106. [CrossRef]

66. Weiss, J.; McClements, D.J.; Takhistov, P. Functional materials in food nanotechnology. J. Food Sci. 2007, 59, 274-275. [CrossRef]

67. Oksman, K.; Mathew, A.P.; Bondeson, D.; Kvien, I. Manufacturing process of cellulose whiskers/polylactic acid nanocomposites. Compos. Sci. Technol. 2006, 66, 2776-2784. [CrossRef]

68. Ray, S.S.; Maiti, P.; Okamoto, M.; Yamada, K.; Ueda, K. New polylactide/layered silicate nanocomposites. 1. Preparation, characterization, and properties. Macromolecules 2002, 35, 3104-3110.

69. Ray, S.S.; Yamada, K.; Okamoto, M.; Ogami, A.; Ueda, K. New polylactide/layered silicate nanocomposites. 3. High-performance biodegradable materials. Chem. Mater. 2003, 15, 1456-1465.

70. Yu, L.; Dean, K.; Li, L. Polymer blends and composites from renewable resources. Prog. Polym. Sci. 2006, 31, 576-602. [CrossRef]

71. Das, K.; Ray, D.; Banerjee, I.; Bandyopadhyay, N.R.; Sengupta, S.; Mohanty, A.K.; Misra, M. Crystalline morphology of PLA/clay nanocomposite films and its correlation with other properties. J. Appl. Polym. Sci. 2010, 118, 143-151. [CrossRef] 
72. Bitinis, N.; Sanz, A.; Nogales, A.; Verdejo, R.; Lopez-Manchado, M.A.; Ezquerra, T.A. Deformation mechanisms in polylactic acid/natural rubber/organoclay bionanocomposites as revealed by synchrotron X-ray scattering. Soft Matter 2012, 8, 8990-8997. [CrossRef]

73. Nofar, M.; Tabatabaei, A.; Park, C.B. Effects of nano-/micro-sized additives on the crystallization behaviors of pla and pla $/ \mathrm{CO}_{2}$ mixtures. Polymer 2013, 54, 2382-2391. [CrossRef]

74. Keshtkar, M.; Nofar, M.; Park, C.B.; Carreau, P.J. Extruded PLA/clay nanocomposite foams blown with supercritical $\mathrm{CO}_{2}$. Polymer 2014, 55, 4077-4090. [CrossRef]

75. Ayana, B.; Suin, S.; Khatua, B.B. Highly exfoliated eco-friendly thermoplastic starch (TPS)/poly(lactic acid)(PLA)/clay nanocomposites using unmodified nanoclay. Carbohydr. Polym. 2014, 110, 430-439.

76. Singh, S.; Ghosh, A.K.; Maiti, S.N.; Raha, S.; Gupta, R.K.; Bhattacharya, S. Morphology and rheological behavior of polylactic acid/clay nanocomposites. Polym. Eng. Sci. 2012, 52, 225-232. [CrossRef]

77. Hapuarachchi, T.D.; Peijs, T. Multiwalled carbon nanotubes and sepiolite nanoclays as flame retardants for polylactide and its natural fiber reinforced composites. Compos. Part A 2010, 41, 954-963. [CrossRef]

78. Busolo, M.A.; Fernandez, P.; Ocio, M.J.; Lagaron, J.M. Novel silver-based nanoclay as an antimicrobial in polylactic acid food packaging coatings. Food Addit. Contam. 2010, 27, 1617-1626. [CrossRef] [PubMed]

79. Meng, Q.K.; Hetzer, M.; De Kee, D. Pla/clay/wood nanocomposites: Nanoclay effects on mechanical and thermal properties. J. Compos. Mater. 2011, 45, 1145-1158. [CrossRef]

80. As'habi, L.; Jafari, S.H.; Khonakdar, H.A.; Boldt, R.; Wagenknecht, U.; Heinrich, G. Tuning the processability, morphology and biodegradability of clay incorporated PLA/LLDPE blends via selective localization of nanoclay induced by melt mixing sequence. Express Polym. Lett. 2013, 7, 21-39. [CrossRef]

81. Lai, S.M.; Hsieh, Y.T. Preparation and properties of polylactic acid (PLA)/silica nanocomposites. J. Macromol. Sci. B 2016, 55, 211-228. [CrossRef]

82. Basilissi, L.; Di Silvestro, G.; Farina, H.; Ortenzi, M.A. Synthesis and characterization of pla nanocomposites containing nanosilica modified with different organosilanes II: Effect of the organosilanes on the properties of nanocomposites: Thermal characterization. J. Appl. Polym. Sci. 2013, 128, 3057-3063. [CrossRef]

83. Mooney, E.; Mackle, J.N.; Blond, D.J.P.; O'Cearbhaill, E.; Shaw, G.; Blau, W.J.; Barry, F.P.; Barron, V.; Murphy, J.M. The electrical stimulation of carbon nanotubes to provide a cardiomimetic cue to mscs. Biomaterials 2012, 33, 6132-6139. [CrossRef] [PubMed]

84. Obarzanek-Fojt, M.; Elbs-Glatz, Y.; Lizundia, E.; Diener, L.; Sarasua, J.R.; Bruinink, A. From implantation to degradation-Are poly (L-lactide)/multiwall carbon nanotube composite materials really cytocompatible? Nanomed. Nanotechnol. 2014, 10, 1041-1051. [CrossRef] [PubMed]

85. Gorrasi, G.; Milone, C.; Piperopoulos, E.; Lanza, M.; Sorrentino, A. Hybrid clay mineral-carbon nanotube-PLA nanocomposite films. Preparation and photodegradation effect on their mechanical, thermal and electrical properties. Appl. Clay Sci. 2013, 71, 49-54. [CrossRef]

86. Supronowicz, P.R.; Ajayan, P.M.; Ullmann, K.R.; Arulanandam, B.P.; Metzger, D.W.; Bizios, R. Novel current-conducting composite substrates for exposing osteoblasts to alternating current stimulation. J. Biomed. Mater. Res. 2002, 59, 499-506. [CrossRef] [PubMed]

87. Kumar, B.; Castro, M.; Feller, J.F. Poly(lactic acid)-multi-wall carbon nanotube conductive biopolymer nanocomposite vapour sensors. Sens. Actuators B 2012, 161, 621-628. [CrossRef]

88. Chiu, W.M.; Chang, Y.A.; Kuo, H.Y.; Lin, M.H.; Wen, H.C. A study of carbon nanotubes/biodegradable plastic polylactic acid composites. J. Appl. Polym. Sci. 2008, 108, 3024-3030. [CrossRef]

89. Novoselov, K.S.; Jiang, D.; Schedin, F.; Booth, T.J.; Khotkevich, V.V.; Morozov, S.V.; Geim, A.K. Two-dimensional atomic crystals. Proc. Natl. Acad. Sci. USA 2005, 102, 10451-10453. [CrossRef] [PubMed]

90. Avouris, P.; Dimitrakopoulos, C. Graphene: Synthesis and applications. Mater. Today 2012, 15, 86-97. [CrossRef]

91. Schwierz, F. Electronics industry-compatible graphene transistors. Nature 2011, 472, 41-42. [CrossRef] [PubMed]

92. Schwierz, F. Graphene transistors. Nat. Nanotechnol. 2010, 5, 487-496. [CrossRef] [PubMed]

93. Schwierz, F. Graphene transistors: Status, prospects, and problems. Proc. IEEE 2013, 101, 1567-1584. [CrossRef]

94. Avouris, P.; Chen, Z.H.; Perebeinos, V. Carbon-based electronics. Nat. Nanotechnol. 2007, 2, 605-615. [CrossRef] [PubMed] 
95. Bao, Q.L.; Loh, K.P. Graphene photonics, plasmonics, and broadband optoelectronic devices. ACS Nano 2012, 6, 3677-3694. [CrossRef] [PubMed]

96. Wang, H.L.; Liang, Y.Y.; Sanchez, H.; Yang, Y.; Cui, L.F.; Cui, Y.; Dai, H.J. Graphene-based hybrid nanomaterials for energy storage applications. Abstr. Pap. Am. Chem. S 2011, 241, 2983-2994.

97. Pumera, M. Graphene-based nanomaterials for energy storage. Energy Environ. Sci. 2011, 4, 668-674. [CrossRef]

98. Radovic, L.R.; Mora-Vilches, C.; Salgado-Casanova, A.J.A. Catalysis: An old but new challenge for graphene-based materials. Chin. J. Catal. 2014, 35, 792-797. [CrossRef]

99. Machado, B.F.; Serp, P. Graphene-based materials for catalysis. Catal. Sci. Technol. 2012, 2, 54-75. [CrossRef]

100. Dikin, D.A.; Stankovich, S.; Zimney, E.J.; Piner, R.D.; Dommett, G.H.B.; Evmenenko, G.; Nguyen, S.T.; Ruoff, R.S. Preparation and characterization of graphene oxide paper. Nature 2007, 448, 457-460. [CrossRef] [PubMed]

101. Bunch, J.S.; Verbridge, S.S.; Alden, J.S.; van der Zande, A.M.; Parpia, J.M.; Craighead, H.G.; McEuen, P.L. Impermeable atomic membranes from graphene sheets. Nano Lett. 2008, 8, 2458-2462. [CrossRef] [PubMed]

102. Katsnelson, M.I. Graphene: Carbon in two dimensions. Mater. Today 2007, 10, 20-27. [CrossRef]

103. Cui, Y.B.; Kundalwal, S.I.; Kumar, S. Gas barrier performance of graphene/polymer nanocomposites. Carbon 2016, 98, 313-333. [CrossRef]

104. Kim, H.; Abdala, A.A.; Macosko, C.W. Graphene/polymer nanocomposites. Macromolecules 2010, 43, 6515-6530. [CrossRef]

105. Feng, L.Z.; Liu, Z.A. Graphene in biomedicine: Opportunities and challenges. Nanomedicine 2011, 6, 317-324. [CrossRef] [PubMed]

106. Lu, C.H.; Yang, H.H.; Zhu, C.L.; Chen, X.; Chen, G.N. A graphene platform for sensing biomolecules. Angew. Chem. Int. Ed. 2009, 48, 4785-4787. [CrossRef] [PubMed]

107. Kuila, T.; Bose, S.; Khanra, P.; Mishra, A.K.; Kim, N.H.; Lee, J.H. Recent advances in graphene-based biosensors. Biosens. Bioelectron. 2011, 26, 4637-4648. [CrossRef] [PubMed]

108. Shao, J.J.; Zheng, D.Y.; Li, Z.J.; Yang, Q.H. Top-down fabrication of two-dimensional nanomaterials: Controllable liquid phase exfoliation. New Carbon Mater. 2016, 31, 97-114.

109. Tang, L.B.; Li, X.M.; Ji, R.B.; Teng, K.S.; Tai, G.; Ye, J.; Wei, C.S.; Lau, S.P. Bottom-up synthesis of large-scale graphene oxide nanosheets. J. Mater. Chem. 2012, 22, 5676-5683. [CrossRef]

110. Zhang, Y.; Zhang, L.Y.; Zhou, C.W. Review of chemical vapor deposition of graphene and related applications. Acc. Chem. Res. 2013, 46, 2329-2339. [CrossRef] [PubMed]

111. Edwards, R.S.; Coleman, K.S. Graphene synthesis: Relationship to applications. Nanoscale 2013, 5, 38-51. [CrossRef] [PubMed]

112. Ciesielski, A.; Samori, P. Graphene via sonication assisted liquid-phase exfoliation. Chem. Soc. Rev. 2014, 43, 381-398. [CrossRef] [PubMed]

113. Vivekanand Prajapati, P.K.S. Arunabha Banik. Carbon nanotubes and its applications. Int. J. Pharm. Sci. Res. 2010, 3, 1099-1107.

114. Terrones, M. Science and technology of the twenty-first century: Synthesis, properties and applications of carbon nanotubes. Annu. Rev. Mater. Res. 2003, 33, 419-501. [CrossRef]

115. De Volder, M.F.L.; Tawfick, S.H.; Baughman, R.H.; Hart, A.J. Carbon nanotubes: Present and future commercial applications. Science 2013, 339, 535-539. [CrossRef] [PubMed]

116. Mamalis, A.G.; Voglander, L.O.G.; Markopoulos, A. Nanotechnology and nanostructured materials: Trends in carbon nanotubes. Precis. Eng. 2004, 28, 16-30. [CrossRef]

117. Zhang, Q.; Huang, J.Q.; Zhao, M.Q.; Qian, W.Z.; Wei, F. Carbon nanotube mass production: Principles and processes. Chemsuschem 2011, 4, 864-889. [CrossRef] [PubMed]

118. Aqel, A.; Abou El-Nour, K.M.M.; Ammar, R.A.A.; Al-Warthan, A. Carbon nanotubes, science and technology part (i) structure, synthesis and characterisation. Arab. J. Chem. 2012, 5, 1-23. [CrossRef]

119. Kumar, M.; Ando, Y. Chemical vapor deposition of carbon nanotubes: A review on growth mechanism and mass production. J. Nanosci. Nanotechnol. 2010, 10, 3739-3758. [CrossRef] [PubMed]

120. Chen, X.P.; Zhang, L.L.; Chen, S.S. Large area cvd growth of graphene. Synth. Met. 2015, 210, 95-108. [CrossRef] 
121. Saito, N.; Usui, Y.; Aoki, K.; Narita, N.; Shimizu, M.; Hara, K.; Ogiwara, N.; Nakamura, K.; Ishigaki, N.; Kato, H.; et al. Carbon nanotubes: Biomaterial applications. Chem. Soc. Rev. 2009, 38, 1897-1903. [CrossRef] [PubMed]

122. Dalton, A.B.; Collins, S.; Razal, J.; Munoz, E.; Ebron, V.H.; Kim, B.G.; Coleman, J.N.; Ferraris, J.P.; Baughman, R.H. Continuous carbon nanotube composite fibers: Properties, potential applications, and problems. J. Mater. Chem. 2004, 14, 1-3. [CrossRef]

123. Wang, J. Carbon-nanotube based electrochemical biosensors: A review. Electroanal 2005, 17, 7-14. [CrossRef]

124. Darkrim, F.L.; Malbrunot, P.; Tartaglia, G.P. Review of hydrogen storage by adsorption in carbon nanotubes. Int. J. Hydrogen Energy 2002, 27, 193-202. [CrossRef]

125. Bonard, J.M.; Kind, H.; Stockli, T.; Nilsson, L.A. Field emission from carbon nanotubes: The first five years. Solid State Electron. 2001, 45, 893-914. [CrossRef]

126. Paradise, M.; Goswami, T. Carbon nanotubes_Production and industrial applications. Mater. Des. 2007, 28, 1477-1489. [CrossRef]

127. Malarkey, E.B.; Parpura, V. Applications of carbon nanotubes in neurobiology. Neurodegener. Dis. 2007, 4, 292-299. [CrossRef] [PubMed]

128. Lacerda, L.; Bianco, A.; Prato, M.; Kostarelos, K. Carbon nanotubes as nanomedicines: From toxicology to pharmacology. Adv. Drug Deliv. Rev. 2006, 58, 1460-1470. [CrossRef] [PubMed]

129. Riehemann, K. Nanotoxicity: How the body develops a way to reduce the toxicity of carbon nanotubes. Small 2012, 8, 1970-1972. [CrossRef] [PubMed]

130. Ren, W.C.; Cheng, H.M. The global growth of graphene. Nat. Nanotechnol. 2014, 9, 726-730. [CrossRef] [PubMed]

131. Si, Y.; Samulski, E.T. Synthesis of water soluble graphene. Nano Lett. 2008, 8, 1679-1682. [CrossRef] [PubMed]

132. Delogu, F.; Gorrasi, G.; Sorrentino, A. Fabrication of polymer nanocomposites via ball milling: Present status and future perspectives. Prog. Mater. Sci. 2017, 86, 75-126. [CrossRef]

133. Moniruzzaman, M.; Winey, K.I. Polymer nanocomposites containing carbon nanotubes. Macromolecules 2006, 39, 5194-5205. [CrossRef]

134. Tait, M.; Pegoretti, A.; Dorigato, A.; Kalaitzidou, K. The effect of filler type and content and the manufacturing process on the performance of multifunctional carbon/poly-lactide composites. Carbon 2011, 49, 4280-4290. [CrossRef]

135. Pinto, A.M.; Cabral, J.; Tanaka, D.A.P.; Mendes, A.M.; Magalhaes, F.D. Effect of incorporation of graphene oxide and graphene nanoplatelets on mechanical and gas permeability properties of poly(lactic acid) films. Polym. Int. 2013, 62, 33-40. [CrossRef]

136. Du, J.H.; Cheng, H.M. The fabrication, properties, and uses of graphene/polymer composites. Macromol. Chem. Phys. 2012, 213, 1060-1077. [CrossRef]

137. Huang, X.; Yin, Z.Y.; Wu, S.X.; Qi, X.Y.; He, Q.Y.; Zhang, Q.C.; Yan, Q.Y.; Boey, F.; Zhang, H. Graphene-based materials: Synthesis, characterization, properties, and applications. Small 2011, 7, 1876-1902. [CrossRef] [PubMed]

138. Moon, S.I.; Jin, F.; Lee, C.; Tsutsumi, S.; Hyon, S.H. Novel carbon nanotube/poly(L-lactic acid) nanocomposites; their modulus, thermal stability, and electrical conductivity. Macromol. Symp. 2005, 224, 287-295. [CrossRef]

139. He, L.H.; Sun, J.; Wang, X.X.; Fan, X.H.; Zhao, Q.L.; Cai, L.F.; Song, R.; Ma, Z.; Huang, W. Unzipped multiwalled carbon nanotubes-incorporated poly(L-lactide) nanocomposites with enhanced interface and hydrolytic degradation. Mater. Chem. Phys. 2012, 134, 1059-1066. [CrossRef]

140. Seligra, P.G.; Nuevo, F.; Lamanna, M.; Fama, L. Covalent grafting of carbon nanotubes to pla in order to improve compatibility. Compos. Part. B Eng. 2013, 46, 61-68. [CrossRef]

141. Yoon, J.T.; Jeong, Y.G.; Lee, S.C.; Min, B.G. Influences of poly(lactic acid)-grafted carbon nanotube on thermal, mechanical, and electrical properties of poly(lactic acid). Polym. Adv. Technol. 2009, 20, 631-638. [CrossRef]

142. Kim, H.S.; Chae, Y.S.; Park, B.H.; Yoon, J.S.; Kang, M.; Jin, H.J. Thermal and electrical conductivity of poly(L-lactide)/multiwalled carbon nanotube nanocomposites. Curr. Appl. Phys. 2008, 8, 803-806. [CrossRef]

143. Yoon, J.T.; Lee, S.C.; Jeong, Y.G. Effects of grafted chain length on mechanical and electrical properties of nanocomposites containing polylactide-grafted carbon nanotubes. Compos. Sci. Technol. 2010, 70, 776-782. [CrossRef] 
144. Chiu, W.M.; Kuo, H.Y.; Tsai, P.A.; Wu, J.H. Preparation and properties of poly (lactic acid) nanocomposites filled with functionalized single-walled carbon nanotubes. J. Polym. Environ. 2013, 21, 350-358. [CrossRef]

145. Kong, Y.X.; Yuan, J.; Wang, Z.M.; Qiu, J. Study on the preparation and properties of aligned carbon nanotubes/polylactide composite fibers. Polym. Compos. 2012, 33, 1613-1619. [CrossRef]

146. Chrissafis, K.; Paraskevopoulos, K.M.; Jannakoudakis, A.; Beslikas, T.; Bikiaris, D. Oxidized multiwalled carbon nanotubes as effective reinforcement and thermal stability agents of poly(lactic acid) ligaments. J. Appl. Polym. Sci. 2010, 118, 2712-2721. [CrossRef]

147. McCullen, S.D.; Stano, K.L.; Stevens, D.R.; Roberts, W.A.; Monteiro-Riviere, N.A.; Clarke, L.I.; Gorga, R.E. Development, optimization, and characterization of electrospun poly(lactic acid) nanofibers containing multi-walled carbon nanotubes. J. Appl. Polym. Sci. 2007, 105, 1668-1678. [CrossRef]

148. Vaisman, L.; Wagner, H.D.; Marom, G. The role of surfactants in dispersion of carbon nanotubes. Adv. Colloid Interface Sci. 2006, 128, 37-46. [CrossRef] [PubMed]

149. Pinto, A.M.; Moreira, S.; Goncalves, I.C.; Gama, F.M.; Mendes, A.M.; Magalhaes, F.D. Biocompatibility of poly(lactic acid) with incorporated graphene-based materials. Colloid Surf. B 2013, 104, 229-238. [CrossRef] [PubMed]

150. Li, W.X.; Xu, Z.W.; Chen, L.; Shan, M.J.; Tian, X.; Yang, C.Y.; Lv, H.M.; Qian, X.M. A facile method to produce graphene oxide-g-poly(L-lactic acid) as an promising reinforcement for plla nanocomposites. Chem. Eng. J. 2014, 237, 291-299. [CrossRef]

151. Li, W.X.; Shi, C.B.; Shan, M.J.; Guo, Q.W.; Xu, Z.W.; Wang, Z.; Yang, C.Y.; Mai, W.; Niu, J.R. Influence of silanized low-dimensional carbon nanofillers on mechanical, thermomechanical, and crystallization behaviors of poly(L-lactic acid) composites_A comparative study. J. Appl. Polym. Sci. 2013, 130, 1194-1202. [CrossRef]

152. Cao, Y.W.; Feng, J.C.; Wu, P.Y. Preparation of organically dispersible graphene nanosheet powders through a lyophilization method and their poly(lactic acid) composites. Carbon 2010, 48, 3834-3839. [CrossRef]

153. Wang, H.S.; Qiu, Z.B. Crystallization behaviors of biodegradable poly(L-lactic acid)/graphene oxide nanocomposites from the amorphous state. Thermochim. Acta 2011, 526, 229-236. [CrossRef]

154. Tong, X.Z.; Song, F.; Li, M.Q.; Wang, X.L.; Chin, I.J.; Wang, Y.Z. Fabrication of graphene/polylactide nanocomposites with improved properties. Compos. Sci. Technol. 2013, 88, 33-38. [CrossRef]

155. Shen, Y.X.; Jing, T.; Ren, W.J.; Zhang, J.W.; Jiang, Z.G.; Yu, Z.Z.; Dasari, A. Chemical and thermal reduction of graphene oxide and its electrically conductive polylactic acid nanocomposites. Compos. Sci. Technol. 2012, 72, 1430-1435. [CrossRef]

156. Huang, H.D.; Ren, P.G.; Xu, J.Z.; Xu, L.; Zhong, G.J.; Hsiao, B.S.; Li, Z.M. Improved barrier properties of poly(lactic acid) with randomly dispersed graphene oxide nanosheets. J. Membr. Sci. 2014, 464, 110-118. [CrossRef]

157. Wu, D.F.; Cheng, Y.X.; Feng, S.H.; Yao, Z.; Zhang, M. Crystallization behavior of polylactide/graphene composites. Ind. Eng. Chem. Res. 2013, 52, 6731-6739. [CrossRef]

158. Sisti, L.; Belcari, J.; Mazzocchetti, L.; Totaro, G.; Vannini, M.; Giorgini, L.; Zucchelli, A.; Celli, A. Multicomponent reinforcing system for poly(butylene succinate): Composites containing poly(L-lactide) electrospun mats loaded with graphene. Polym. Test. 2016, 50, 283-291. [CrossRef]

159. Verdejo, R.; Bernal, M.M.; Romasanta, L.J.; Lopez-Manchado, M.A. Graphene filled polymer nanocomposites. J. Mater. Chem. 2011, 21, 3301-3310. [CrossRef]

160. Lin, W.Y.; Shih, Y.F.; Lin, C.H.; Lee, C.C.; Yu, Y.H. The preparation of multi-walled carbon nanotube/poly(lactic acid) composites with excellent conductivity. J. Taiwan Inst. Chem. E 2013, 44, 489-496. [CrossRef]

161. Barrau, S.; Vanmansart, C.; Moreau, M.; Addad, A.; Stoclet, G.; Lefebvre, J.M.; Seguela, R. Crystallization behavior of carbon nanotube-polylactide nanocomposites. Macromolecules 2011, 44, 6496-6502. [CrossRef]

162. Villmow, T.; Potschke, P.; Pegel, S.; Haussler, L.; Kretzschmar, B. Influence of twin-screw extrusion conditions on the dispersion of multi-walled carbon nanotubes in a poly(lactic acid) matrix. Polymer 2008, 49, 3500-3509. [CrossRef]

163. Kuan, C.F.; Kuan, H.C.; Ma, C.C.M.; Chen, C.H. Mechanical and electrical properties of multi-wall carbon nanotube/poly(lactic acid) composites. J. Phys. Chem. Solids 2008, 69, 1395-1398. [CrossRef] 
164. Lei, L.; Qiu, J.H.; Sakai, E. Preparing conductive poly(lactic acid) (PLA) with poly(methyl methacrylate) (PMMA) functionalized graphene (PFG) by admicellar polymerization. Chem. Eng. J. 2012, 209, 20-27. [CrossRef]

165. Gorrasi, G.; Sorrentino, A. Photo-oxidative stabilization of carbon nanotubes on polylactic acid. Polym. Degrad. Stab. 2013, 98, 963-971. [CrossRef]

166. Ali, A.M.; Ahmad, S.H. Mechanical characterization and morphology of polylactic acid/liquid natural rubber filled with multi walled carbon nanotubes. AIP Conf. Proc. 2013, 1571, 83-89.

167. Chieng, B.W.; Ibrahim, N.A.; Yunus, W.M.Z.W.; Hussein, M.Z. Poly(lactic acid)/poly(ethylene glycol) polymer nanocomposites: Effects of graphene nanoplatelets. Polymers 2014, 6, 93-104. [CrossRef]

168. Bao, C.L.; Song, L.; Xing, W.Y.; Yuan, B.H.; Wilkie, C.A.; Huang, J.L.; Guo, Y.Q.; Hu, Y. Preparation of graphene by pressurized oxidation and multiplex reduction and its polymer nanocomposites by masterbatch-based melt blending. J. Mater. Chem. 2012, 22, 6088-6096. [CrossRef]

169. Ali, A.M.; Ahmad, S.H. Effect of processing parameter and filler content on tensile properties of multi-walled carbon nanotubes reinforced polylactic acid nanocomposite. In Proceedings of the 2012 National Physics Conference, PERFIK 2012, Bukit Tinggi, Malaysia, 19-21 Novembwr 2012; pp. 254-259.

170. Kim, S.Y.; Shin, K.S.; Lee, S.H.; Kim, K.W.; Youn, J.R. Unique crystallization behavior of multi-walled carbon nanotube filled poly(lactic acid). Fibers Polym. 2010, 11, 1018-1023. [CrossRef]

171. Murariu, M.; Dechief, A.L.; Bonnaud, L.; Paint, Y.; Gallos, A.; Fontaine, G.; Bourbigot, S.; Dubois, P. The production and properties of polylactide composites filled with expanded graphite. Polym. Degrad. Stab. 2010, 95, 889-900. [CrossRef]

172. Hassouna, F.; Laachachi, A.; Chapron, D.; El Mouedden, Y.; Toniazzo, V.; Ruch, D. Development of new approach based on raman spectroscopy to study the dispersion of expanded graphite in poly(lactide). Polym. Degrad. Stab. 2011, 96, 2040-2047. [CrossRef]

173. Potts, J.R.; Dreyer, D.R.; Bielawski, C.W.; Ruoff, R.S. Graphene-based polymer nanocomposites. Polymer 2011, 52, 5-25. [CrossRef]

174. Kim, H.; Kobayashi, S.; AbdurRahim, M.A.; Zhang, M.L.J.; Khusainova, A.; Hillmyer, M.A.; Abdala, A.A.; Macosko, C.W. Graphene/polyethylene nanocomposites: Effect of polyethylene functionalization and blending methods. Polymer 2011, 52, 1837-1846. [CrossRef]

175. Brzeziński, M.; Biela, T. Polylactide nanocomposites with functional carbon nanotubes: A focused review. Mater. Lett. 2014, 244-250. [CrossRef]

176. Yang, J.H.; Lin, S.H.; Lee, Y.D. Preparation and characterization of poly(L-lactide)-graphene composites using the in situ ring-opening polymerization of plla with graphene as the initiator. J. Mater. Chem. 2012, 22, 10805-10815. [CrossRef]

177. Pramoda, K.P.; Koh, C.B.; Hazrat, H.; He, C.B. Performance enhancement of polylactide by nanoblending with poss and graphene oxide. Polym. Compos. 2014, 35, 118-126. [CrossRef]

178. Li, Q.H.; Zhou, Q.H.; Deng, D.; Yu, Q.Z.; Gu, L.; Gong, K.D.; Xu, K.H. Enhanced thermal and electrical properties of poly (D,L-lactide)/multi-walled carbon nanotubes composites by in-situ polymerization. Trans. Nonferr. Met. Soc. China 2013, 23, 1421-1427. [CrossRef]

179. Yoon, O.J.; Sohn, I.Y.; Kim, D.J.; Lee, N.E. Enhancement of thermomechanical properties of poly(D,L-lactic-co-glycolic acid) and graphene oxide composite films for scaffolds. Macromol. Res. 2012, 20, 789-794. [CrossRef]

180. Pinto, A.M.; Goncalves, C.; Goncalves, I.C.; Magalhaes, F.D. Effect of biodegradation on thermo-mechanical properties and biocompatibility of poly(lactic acid)/graphene nanoplatelets composites. Eur. Polym. J. 2016, 85, 431-444. [CrossRef]

181. Desa, M.S.Z.M.; Hassan, A.; Arsad, A. The effect of natural rubber toughening on mechanical properties of poly(lactic acid)/multiwalled carbon nanotube nanocomposite. Adv. Mater. Res. 2013, 747, 639-642. [CrossRef]

182. Goncalves, C.; Pinto, A.; Machado, A.V.; Moreira, J.A.; Gonçalves, I.C.; Magalhães, F.D. Biocompatible reinforcement of poly(lactic acid) with graphene nanoplatelets. Polym. Compos. 2016. [CrossRef]

183. Shao, S.J.; Zhou, S.B.; Li, L.; Li, J.R.; Luo, C.; Wang, J.X.; Li, X.H.; Weng, J. Osteoblast function on electrically conductive electrospun pla/mwcnts nanofibers. Biomaterials 2011, 32, 2821-2833. [CrossRef] [PubMed] 
184. Potschke, P.; Andres, T.; Villmow, T.; Pegel, S.; Brunig, H.; Kobashi, K.; Fischer, D.; Haussler, L. Liquid sensing properties of fibers prepared by melt spinning from poly(lactic acid) containing multi-walled carbon nanotubes. Compos. Sci. Technol. 2010, 70, 343-349. [CrossRef]

185. Ma, H.B.; Su, W.X.; Tai, Z.X.; Sun, D.F.; Yan, X.B.; Liu, B.; Xue, Q.J. Preparation and cytocompatibility of polylactic acid/hydroxyapatite/graphene oxide nanocomposite fibrous membrane. Chin. Sci. Bull. 2012, 57, 3051-3058. [CrossRef]

186. Magrez, A.; Kasas, S.; Salicio, V.; Pasquier, N.; Seo, J.W.; Celio, M.; Catsicas, S.; Schwaller, B.; Forro, L. Cellular toxicity of carbon-based nanomaterials. Nano Lett. 2006, 6, 1121-1125. [CrossRef] [PubMed]

187. McCullen, S.D.; Stevens, D.R.; Roberts, W.A.; Clarke, L.I.; Bernacki, S.H.; Gorga, R.E.; Loboa, E.G. Characterization of electrospun nanocomposite scaffolds and biocompatibility with adipose-derived human mesenchymal stem cells. Int. J. Nanomed. 2007, 2, 253-263.

188. Sherrell, P.C.; Thompson, B.C.; Wassei, J.K.; Gelmi, A.A.; Higgins, M.J.; Kaner, R.B.; Wallace, G.G. Maintaining cytocompatibility of biopolymers through a graphene layer for electrical stimulation of nerve cells. Adv. Funct. Mater. 2014, 24, 769-776. [CrossRef]

189. Li, Z.Q.; Zhao, X.W.; Ye, L.; Coates, P.; Caton-Rose, F.; Martyn, M. Structure and blood compatibility of highly oriented PLA/mwnts composites produced by solid hot drawing. J. Biomater. Appl. 2014, 28, 978-989. [CrossRef] [PubMed]

190. An, X.L.; Ma, H.B.; Liu, B.; Wang, J.Z. Graphene oxide reinforced polylactic acid/polyurethane antibacterial composites. J. Nanomater. 2013, 2013. [CrossRef]

191. Yoon, O.J.; Jung, C.Y.; Sohn, I.Y.; Kim, H.J.; Hong, B.; Jhon, M.S.; Lee, N.E. Nanocomposite nanofibers of poly(D,L-lactic-co-glycolic acid) and graphene oxide nanosheets. Compos. Part A 2011, 42, 1978-1984. [CrossRef]

192. Kanczler, J.M.; Mirmalek-Sani, S.H.; Hanley, N.A.; Ivanov, A.L.; Barry, J.J.A.; Upton, C.; Shakesheff, K.M.; Howdle, S.M.; Antonov, E.N.; Bagratashvili, V.N.; et al. Biocompatibility and osteogenic potential of human fetal femur-derived cells on surface selective laser sintered scaffolds. Acta Biomater. 2009, 5, 2063-2071. [CrossRef] [PubMed]

193. Pinto, V.C.; Costa-Almeida, R.; Rodrigues, I.; Guardão, L.; Soares, R.; Guedes, R.M. Biocompatibility of PLA/GNP and PLA/CNT-cooh nanocomposites. J. Biomed. Mater. Res. Part A 2017, 105A, 2182-2190. [CrossRef] [PubMed] 